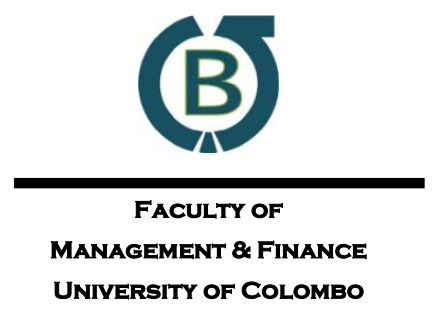

\title{
The Impact of Transformational Leadership on Employee Well-being: A Narrative Synthesis
}

\author{
Chaturi Pilane Liyanage $\mathbf{a}$ \\ ${ }^{a}$ Coventry Business School, University of Coventry, UK
}

\begin{abstract}
Despite the growing popularity of the construct of Transformational leadership, there is little consensus and understanding on the socio-psychological process that facilitates the relationship between Transformational leadership and employee well-being. To address this gap in the literature a systematic analysis of 30 empirical research studies was conducted, guided by the primary review question, "how does Transformational leadership influence employee well-being?" The synthesis revealed that Transformational leadership predicts employee well-being through several socio-psychological mechanisms. Notably, meaningful work, perceived procedural justice, psychological empowerment, self and team efficacy, empowerment, psychological resources, trust, perception of work-life conflict and role clarity were identified as mediating the said relationship, and power distance, employee ambition and organisation commitment were identified as moderators. It is believed that introducing leadership training in the identified socio-psychological sources would result in a productive occupational health intervention, thus instigating a collaborative and healthy organisational culture built on employee well-being.
\end{abstract}

Keywords: Transformational Leadership, Employee Well-being, Psychological Wellbeing, Systematic Literature Review

Received:

31 May 2020
Accepted revised version: 18 August 2020
Published:

31 December 2020

Suggested citation: Liyanage, C. P. (2020). The impact of transformational leadership on employee well-being: A narrative synthesis. Colombo Business Journal. 11(2), 24-66.

DOI: http://doi.org/10.4038/cbj.v11i2.63

(C) 2020 The Author. This work is licensed under a Creative Commons Attribution 4.0 International License which permits unrestricted use, distribution, and reproduction in any medium, provided the original work is properly cited.

\ chatu.0026@gmail.com: (iD https://orcid.org/0000-0001-7553-1998 


\section{Introduction}

In the past decade Transformational leadership has gained considerable attention over other forms of leadership styles (Judge \& Bono, 2000). In fact, numerous research studies have been conducted in different settings ranging from corporate organisations to healthcare organisations in order to ascertain the impact of Transformational leadership across different contextual settings. Interestingly, with the increase in attention towards employee well-being, researchers have engaged in empirical research to identify the role played by Transformational leaders in instigating well-being, job commitment and job satisfaction within teams (Medley \& Larochelle, 1995; Neilsen, Randall et al., 2008; Sofarelli \& Brown, 1998; Thyer, 2003).

However, in management research there is a lack of comprehensive understanding regarding the impact of Transformational leadership on different dimensions of employee well-being. Thus, the purpose of this study is to systematically analyse and synthesise the current body of research that have explored the relationship between Transformational leadership and employee well-being. In this review empirical studies that investigated Transformational leadership in an organisational setting were considered and studies revolving around leadership in other settings such as sports, for example, were excluded. Earlier reviews on this topic have focused on the impact of leadership on well-being without a specific focus on Transformational leadership (Arnold \& Connelly, 2015; Clarke et al., 2015). Although there was one review that explored the relationship between the two constructs, it broadly conceptualised employee well-being as psychological wellbeing while ignoring the impact of Transformational leadership on other dimensions of well-being such as perceived strain (Arnold, 2017). Thus, it is believed that by using the overarching construct of well-being, this review captured the impact of Transformational leadership on different dimensions of well-being (of which psychological well-being is just one dimension).

In order to address the lacunae in research illustrated above, the aim of this systematic literature review is to present the findings in a form of a narrative synthesis that focuses on the primary research question, how does Transformational leadership influence employee well-being? In order to find the answer to this research question, two secondary research objectives were outlined, namely: (1) to understand if Transformational leadership predicts employee well-being in different organisational contexts, and (2) to decode how certain psychological processes act as a mediator or a moderator in facilitating or strengthening the relationship between Transformational leadership and employee well-being. 
It is believed that the aforementioned research question(s) and objectives will help to fill the previously identified gap in the existing research as only a few research studies have adopted a descriptive analytical approach that decodes the psychological process between Transformational leadership and employee well-being (Nielsen et al., 2009).

This paper will first provide an overview of the constructs of leadership, wellbeing and the relationship between the two. Thereafter, the methodological approach adopted for the synthesis of evidence is explained. The psychological processors underpinning the relationship between Transformational leadership and well-being will be explored in detail in the proceeding section, where the findings of the review are analysed, and implications of the analysis will be outlined accordingly. In the last section a reflection on the synthesis of evidence generated and directions for future research will be suggested.

\section{Transformational Leadership Decoded}

Transformational leadership has the ability to inspire followers to make a contribution to one's organisation and the community at large. As defined by Pearce and Conger (2003), Transformational leadership, that goes beyond incentives for performance, is said to encourage and develop workers intellectually and creatively, while integrating employee concern as an integral aspect of the company's mission. Echoing the same sentiment Trofino (2000) asserts that Transformational leaders set a clear vision for their organisation. In fact, all research papers defined the concept of Transformational leadership consistently, adopting the conceptual framework presented by Bass (1985), where Transformational leadership is often defined based on four distinct dimensions, namely idealised influence, inspirational motivation, individualised consideration and intellectual stimulation. However, only a few studies evaluated the impact of each element on employee well-being (Franke \& Felfe, 2011; Krishnan, 2012).

The first dimension, idealised influence reflects behaviours where the leaders take initiative based on what is right than what is more lucrative. Leaders are guided by such behaviour due to their deep-rooted moral commitment to both their own actions and to their employees (Turner et al., 2002). Employees in turn will respect and trust such Transformational leaders who engage in behaviour that reflect idealised influence. In fact, due to the desirable qualities possessed by transformational leaders, followers may even wish to emulate such leaders (Bass \& Avolio, 1993). The next characteristic, inspirational motivation is where the leader motivates the followers to 
reach their full potential, by optimistically painting the future for the followers (Bass $\&$ Riggio, 2006). By doing so the leader encourages followers to reach goals that the followers themselves do not consider as possible. By instilling positive feelings of a possible bright future, the Transformational leader looks to inspire and motivate his followers. This leads to the third characteristic of Transformational leadership, individualised consideration, where the leader will act as a mentor to his/her employees and will engage in two-way communications in which the leader will listen and empathise with the followers' experiences (Bass \& Riggio, 2006). It is through individualised consideration that the relationship is cemented between the leader and the follower. The fourth characteristic, intellectual stimulation, is a crucial element in the development stage of an employee as it is this characteristic of the Transformational leader that encourages the follower to question the status quo. As employees are now encouraged to take their own initiative, they will become more confident, in turn, enhancing employee self-efficacy. As such, this paper will explore how the four dimensions of Transformational leadership influence employee wellbeing.

\section{Employee Well-being}

The International Labour Organisation (ILO) (2010) defines employee wellbeing as a criterion that relates to all aspects of working life, "from the quality and safety of the physical environment, to how the employees feels about their work, their working environment, the climate at work and work organisation" (p. 11). However, unlike Transformational leadership, employee well-being is operationalised in numerous ways, ranging from subjective well-being, psychological strain, satisfaction, burnout and the intention to leave (Page \& Vella-Brodrick, 2009; Robertson \& Cooper, 2010). Psychological well-being, which is the most widely discussed construct in relation to well-being is defined by The Organisation for Economic Co-operation and Development (OECD) (2011) as "the various evaluations, positive and negative that people make of their life and the affective reactions of people to their experiences." (p. 11).

Building on this notion, Robertson and Cooper (2010) categorised psychological well-being into two main areas namely Hedonic and Eudaimonic well-being. The Hedonist school focuses on the maximisation of pleasure over pain (Deci \& Ryan, 2008), while Eudaimonic well-being focuses on living a fulfilled life by finding meaning and purpose in one's life. Thus, Eudaimonic well-being is often characterised by personal growth and self-realisation (Deci \& Ryan, 2008). 
The 30 research articles discussed in this systematic review operationalised employee well-being broadly into six areas, namely, Psychological well-being (e.g. Eudaimonic and Hedonic well-being), Affective well-being (e.g. organisation commitment, job satisfaction), Professional well-being (e.g. aspiration and competence at work, autonomy), Social well-being (e.g. good relationships with colleagues), Cognitive well-being (e.g. ability to concentrate at work) and Psychomatic well-being (e.g. health complaints like headaches and stomach aches see Table 1 for a complete overview of the studies).

\section{Methodology}

This systematic literature review adopted a five-step narrative synthesis approach based on the principles outlined by Briner and Denyer (2012). These steps include planning; structured search; evaluating material against agreed criteria; analysis and thematic coding; and reporting. The subject of Transformational leadership and its relationship to employee well-being seems to have been widely researched and has reached a sufficient stage of maturity, therein warranting a synthesis of the current research evidence. It is expected that such an exercise will provide a holistic understanding of the impact of Transformational leadership on employee well-being.

\section{Search Methods}

Published, peer reviewed studies were identified predominantly through electronic sources. The four data bases included in this review are Scopus, Research Gate, Science Direct and EBSCO Business source complete. Only peer reviewed journal articles written in English were sourced. The search for studies was conducted in systematic and pre-determined manner, according to the order of the information sources listed above.

\section{Inclusion, Exclusion Criteria and Search Strategy}

The initial phases of the desk research were conducted using the key search term 'Transformational leadership', and the number of articles containing this leadership construct was recoded. During this stage, no restriction was placed on the date and geographical location of the studies.

During the second stage a rigorous search was conducted by adopting a predetermined search strategy to further narrow down the articles revolving around the subject area of Transformational leadership. The following search terms (with wild cards when required) were used when searching articles from the electronic databases; Transformational (leadership or leader\$ or chief or head\$ or CEO $\$$ or 
Managing Director\$) and employee or staff or worker (well-being or Hedonic wellbeing or Eudaimonic well-being or Psychological well-being or health or cognitive well-being or Psychomatic well-being or Professional well-being or Affective wellbeing or social well-being). If there were duplicates among the data bases it was recorded and deleted accordingly.

Once all relevant research studies pertaining to the defined research question were identified a second screening was conducted in order to ascertain if the papers fulfilled the inclusion criteria. If they did, the full text papers were sourced to be included in the systematic review. The pre-determined inclusion criteria for the second screening of the articles are as follows: (a) The research papers should be in the English language, (b) should exclusively adopt an empirical study (i.e. books, websites, reviews and editorial articles were excluded), and (c) should discuss Transformational leadership as one of the main themes, where no restrictions were imposed on the sample studied, as the intention is to understand how Transformational leadership influences employee's well-being, in different organisational settings. Articles were excluded if the research studies did not meet the stipulated inclusion criteria. If any articles in the bibliographies of selected articles met the inclusion criteria, they too were included in the systematic literature review.

\section{Sample}

The initial open search of the key word Transformational leadership produced 305 items in total across the four databases. However, during the second stage when the delineated search strategy was adopted the research base on Transformational leadership narrowed down to 70 items. After duplicate records were removed only 55 articles remained. Next the titles and abstracts were screened for relevancy for the selected research topic. Based on the abstracts and titles the relevant research articles were categorised under certain pre-specified areas, namely, grey areas, research articles which deal with multiple leadership styles, qualitative and quantitative studies. The articles classified under each folder were extracted for a detailed evaluation. During this process 15 articles were excluded from the study because they were unrelated, for example, most of those studies discussed the impact of Transformational leadership on work life balance rather than discussing its link to employee well-being. Finally, the full text articles of the remaining 40 articles were screened based on the stipulated inclusion and exclusion criteria. Although the complete quality assessment outlined in Appendix 1 was not conducted for all papers (due to time constraints), the quality and study design of all studies were ascertained. For instance, an analysis was conducted to identify if the data collection methods 
adopted were reliable and valid; by doing this application bias was curtailed to a great extent (Briner \& Denyer, 2012). The applications of such rigorous criteria lead to a final pool of 30 empirical research papers to be included in the systematic literature review. A PRISMA flowchart outlined in Figure 1 summarises the systematic process by which the research articles were obtained.

\section{Figure 1: PRISMA Flow Chart}

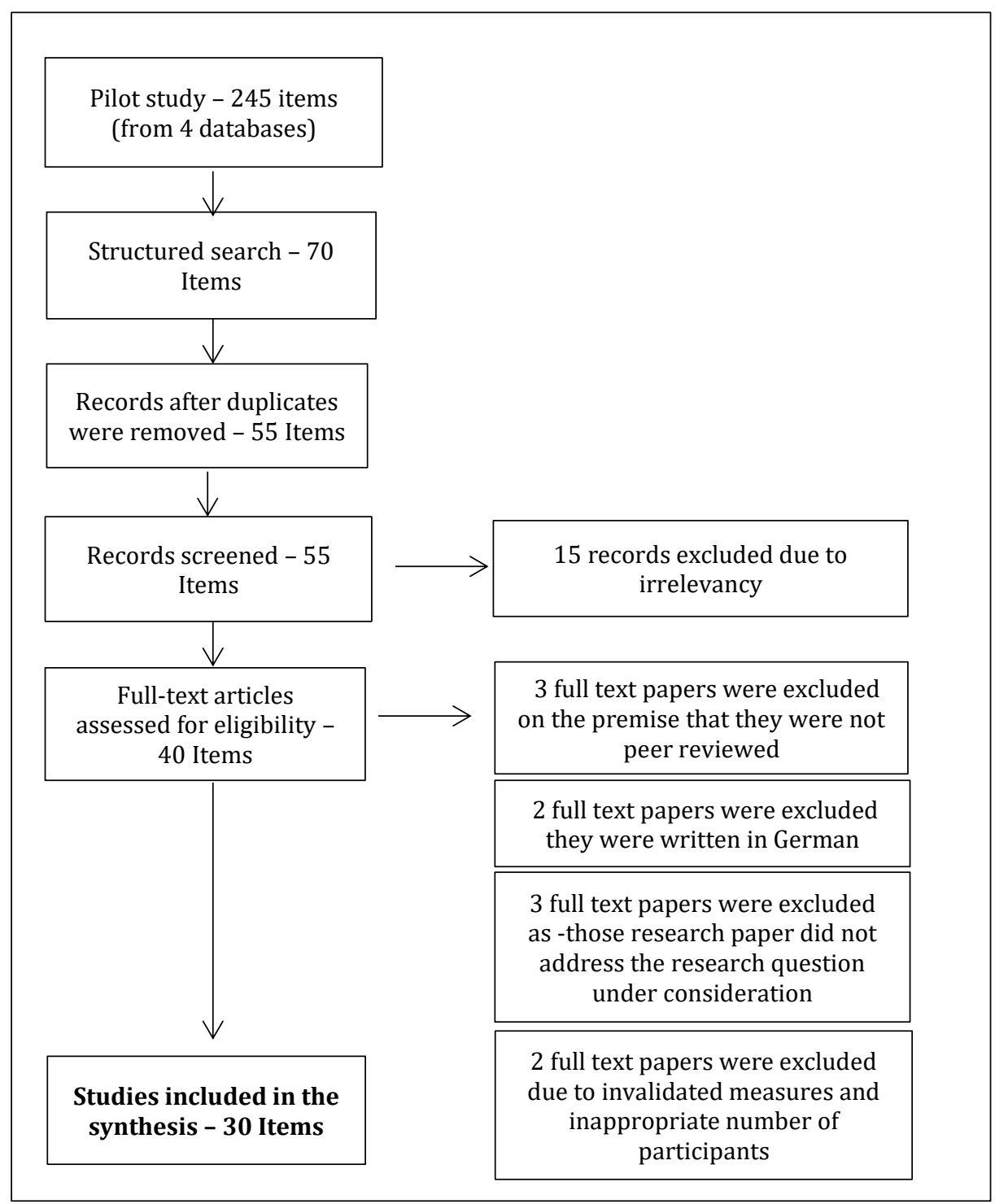

Source: Adapted from Boland et al. (2017) 
It is important to note that the delineated search strategy was implemented during the month of January 2019, hence, papers that were published on this topic after this date are not captured. However, during the time of writing this paper the search strategy adopted previously was repeated to identify any new changes pertaining to the subject area under consideration. As there have not been major changes to the research body, the researcher is confident that the review has captured the key papers revolving around the area of Transformational leadership and employee well-being. The selected journal articles were grouped pertaining to the area of focus. The research studies dealt with multitude of fields, namely, manufacturing (2), education (2), health care (10), business (4), legal enforcement (1), hospitality industry (4), financial sector (2), information technology and communication (2), and non-profit organisations (3). All studies were quantitative in nature.

\section{Data Extraction and Analysis}

The approach adopted in data analysis resembles the data synthesising mechanism suggested by Popay et al. (2006) which indicates that a narrative synthesis should draw connections and patterns among the extracted data of the studies.

As indicated in the data extraction table outlined in the results section, the following key information was obtained from each article (a) What were the mediating/moderating factors between Transformational leadership and employee well-being? (b) In what contexts was the impact of Transformational leadership on employee well-being observed? (c) How was the relationship between the two constructs examined? (d) What were the results of the publication? Finally, as mentioned earlier the methodology adopted to ascertain the relationship between Transformational leadership and employee well-being was validated using quantitative assessment tools. As outlined in Appendix 1 the quantitative quality assessment tool devised by Letts et al. (2007), was adopted to determine the quality of the quantitative studies used in the systematic review. Moreover, a three-point Likert scale was used to identify and eliminate poor quality studies from the consideration pool. This classification is also presented in Appendix 1.

The findings from 30 research papers are summarised in table format (See Table 1) to demonstrate the connections between the variables under consideration. Moreover, a conceptual framework/model was also developed in order to illustrate the multiple processes through which Transformational leadership contributes to employee well-being. 
Table 1: Extraction Table

\begin{tabular}{|c|c|c|c|c|c|c|c|}
\hline \multirow[b]{2}{*}{ Author } & \multirow[b]{2}{*}{ Study Design } & \multicolumn{4}{|c|}{ Variables } & \multirow[b]{2}{*}{ Moderators } & \multirow[b]{2}{*}{ Findings } \\
\hline & & Sample & Predictor Variables & $\begin{array}{l}\text { Outcome } \\
\text { Variables }\end{array}$ & Mediators & & \\
\hline \multirow[t]{2}{*}{$\begin{array}{l}\text { Arnold et } \\
\text { al. (2007) }\end{array}$} & Cross sectional & $\begin{array}{l}\text { Study 1: } 319 \\
\text { healthcare workers }\end{array}$ & $\begin{array}{l}\text { Transformational } \\
\text { leadership }\end{array}$ & $\begin{array}{l}\text { Context free } \\
\text { psychological } \\
\text { wellbeing }\end{array}$ & Meaningful work & None & $\begin{array}{l}\text { Study 1: Meaningful work partially } \\
\text { mediated the relationship between } \\
\text { Transformational leadership and Well- } \\
\text { being }\end{array}$ \\
\hline & & $\begin{array}{l}\text { Study 2:146 } \\
\text { Canadian funeral } \\
\text { directors and dental } \\
\text { hygienists }\end{array}$ & & & & & $\begin{array}{l}\text { Study 2: Meaningful work fully } \\
\text { mediated the relationship between } \\
\text { Transformational leadership and Well- } \\
\text { being }\end{array}$ \\
\hline $\begin{array}{l}\text { Bono et al. } \\
(2007)\end{array}$ & Cross sectional & $\begin{array}{l}57 \text { health care } \\
\text { workers }\end{array}$ & $\begin{array}{l}\text { Transformational } \\
\text { leadership }\end{array}$ & $\begin{array}{l}\text { Affective well- } \\
\text { being }\end{array}$ & None & None & $\begin{array}{l}\text { Transformational leadership was } \\
\text { positively related to enhanced positive } \\
\text { emotions at work and no reports on } \\
\text { the negative emotions. }\end{array}$ \\
\hline $\begin{array}{l}\text { Corrigan et } \\
\text { al. (2002) }\end{array}$ & Cross sectional & $\begin{array}{l}620 \text { mental health } \\
\text { employees in } 54 \\
\text { teams }\end{array}$ & $\begin{array}{l}\text { Transformational } \\
\text { leadership }\end{array}$ & $\begin{array}{l}\text { Psychological } \\
\text { well-being }\end{array}$ & None & None & $\begin{array}{l}\text { Idealised influence, inspirational } \\
\text { motivation individual consideration in } \\
\text { leaders were significantly negatively } \\
\text { related to emotional exhaustion. } \\
\text { Intellectual stimulation, inspirational } \\
\text { motivation and individual } \\
\text { consideration significantly positively } \\
\text { correlated with personal } \\
\text { accomplishment }\end{array}$ \\
\hline $\begin{array}{l}\text { Densten } \\
(2005)\end{array}$ & Cross sectional & $\begin{array}{l}480 \text { senior managers } \\
\text { in an Australian law } \\
\text { enforcement } \\
\text { organisation }\end{array}$ & $\begin{array}{l}\text { Transformational } \\
\text { leadership }\end{array}$ & $\begin{array}{l}\text { Employee well- } \\
\text { being }\end{array}$ & None & None & $\begin{array}{l}\text { Negative relationship between concept } \\
\text { based inspirational motivation and } \\
\text { emotional exhaustion and no } \\
\text { relationship with image based } \\
\text { inspirational motivation. }\end{array}$ \\
\hline
\end{tabular}




\begin{tabular}{|c|c|c|c|c|c|c|c|}
\hline \multirow[b]{2}{*}{ Author } & \multirow[b]{2}{*}{ Study Design } & \multicolumn{4}{|c|}{ Variables } & \multirow[b]{2}{*}{ Moderators } & \multirow[b]{2}{*}{ Findings } \\
\hline & & Sample & Predictor Variables & $\begin{array}{l}\text { Outcome } \\
\text { Variables }\end{array}$ & Mediators & & \\
\hline $\begin{array}{l}\text { Franke and } \\
\text { Felfe } \\
(2011)\end{array}$ & Cross sectional & $\begin{array}{l}710 \text { German } \\
\text { employees working } \\
\text { in public and private } \\
\text { organisations (S1- } \\
526 \text { employees of a } \\
\text { manufacturing } \\
\text { company+ S2-509 } \\
\text { employees in } \\
\text { clerical workers } \\
\text { from public } \\
\text { administration). }\end{array}$ & $\begin{array}{l}\text { 1. Idealised influence } \\
\text { (II) (behaviour) } \\
\text { 2. Idealised influence } \\
\text { (II) (attribute) } \\
\text { 3. Inspirational } \\
\text { motivation (IM) } \\
\text { 4. Intellectual } \\
\text { stimulation (IS) } \\
\text { 5. Individualised } \\
\text { consideration (IC) }\end{array}$ & Perceived strain & None & $\begin{array}{l}\text { Organisational } \\
\text { commitment }\end{array}$ & $\begin{array}{l}\text { IC and II (attribute) appeared to be the } \\
\text { most important predictors, whereas } \\
\text { IM and II (behaviour) did not explain } \\
\text { incremental variance in strain. } \\
\text { Moreover, the relationship between } \\
\text { leader's IC and II (attribute) and } \\
\text { perceived strain is moderated by } \\
\text { affective Organisation commitment. }\end{array}$ \\
\hline $\begin{array}{l}\text { Gill et al. } \\
(2009)\end{array}$ & Cross sectional & $\begin{array}{l}147 \text { hospitality } \\
\text { workers in Canada }\end{array}$ & $\begin{array}{l}\text { Transformational } \\
\text { leadership }\end{array}$ & $\begin{array}{l}\text { Employee well- } \\
\text { being }\end{array}$ & None & None & $\begin{array}{l}\text { Transformational leadership } \\
\text { negatively associated with job stress, } \\
\text { and job stress was positively } \\
\text { associated with burnout. }\end{array}$ \\
\hline $\begin{array}{l}\text { Gill et al. } \\
(2010)\end{array}$ & Cross sectional & $\begin{array}{l}266 \text { hospitality } \\
\text { workers in India }\end{array}$ & $\begin{array}{l}\text { Transformational } \\
\text { leadership }\end{array}$ & $\begin{array}{l}\text { Employee well- } \\
\text { being }\end{array}$ & None & None & $\begin{array}{l}\text { No significant relationship between } \\
\text { Transformational leadership and Job } \\
\text { stress }\end{array}$ \\
\hline $\begin{array}{l}\text { Green et al. } \\
\text { (2014) }\end{array}$ & Cross sectional & $\begin{array}{l}322 \text { clinical and } \\
\text { case management } \\
\text { mental health } \\
\text { service providers in } \\
\text { San Diego }\end{array}$ & $\begin{array}{l}\text { Transformational } \\
\text { leadership }\end{array}$ & $\begin{array}{l}\text { Employee well- } \\
\text { being }\end{array}$ & None & None & $\begin{array}{l}\text { Transformational leadership was } \\
\text { significantly positively related to } \\
\text { increased personal accomplishment }\end{array}$ \\
\hline $\begin{array}{l}\text { Holstad et } \\
\text { al. (2014) }\end{array}$ & Cross sectional & $\begin{array}{l}199 \text { German } \\
\text { employees from } \\
\text { financial and service } \\
\text { sectors }\end{array}$ & $\begin{array}{l}\text { Transformational } \\
\text { leadership }\end{array}$ & $\begin{array}{l}\text { Emotional } \\
\text { strain }\end{array}$ & Social support & $\begin{array}{l}\text { Follower } \\
\text { ambition }\end{array}$ & $\begin{array}{l}\text { Findings were twofold: } \\
\text { 1. Transformational leadership and } \\
\text { follower emotional strain mediated } \\
\text { by social support for medium and } \\
\text { highly ambitious employees } \\
\text { 2. Professional ambition moderated } \\
\text { the mediated relationship. }\end{array}$ \\
\hline
\end{tabular}




\begin{tabular}{|c|c|c|c|c|c|c|c|}
\hline \multirow[b]{2}{*}{ Author } & \multirow[b]{2}{*}{ Study Design } & \multicolumn{4}{|c|}{ Variables } & \multirow[b]{2}{*}{ Moderators } & \multirow[b]{2}{*}{ Findings } \\
\hline & & Sample & Predictor Variables & $\begin{array}{l}\text { Outcome } \\
\text { Variables }\end{array}$ & Mediators & & \\
\hline $\begin{array}{l}\text { Jacobs et } \\
\text { al. (2013) }\end{array}$ & $\begin{array}{l}\text { Online cross } \\
\text { sectional study }\end{array}$ & $\begin{array}{l}318 \text { employees in } \\
\text { German information } \\
\text { and communication } \\
\text { technology (ICT) } \\
\text { organisation }\end{array}$ & $\begin{array}{l}\text { Transformational } \\
\text { leadership }\end{array}$ & Well-being & None & None & $\begin{array}{l}\text { Transformational leadership in ICT is } \\
\text { associated with greater employee } \\
\text { well-being }\end{array}$ \\
\hline $\begin{array}{l}\text { Kara et al. } \\
(2013)\end{array}$ & Cross sectional & $\begin{array}{l}443 \text { employees } \\
\text { in five-star hotels } \\
\text { in Turkey }\end{array}$ & $\begin{array}{l}\text { Transformational } \\
\text { leadership }\end{array}$ & Well-being & $\begin{array}{l}\text { Quality of } \\
\text { working life }\end{array}$ & None & $\begin{array}{l}\text { Transformational leadership and } \\
\text { employee well-being mediated by } \\
\text { quality of working life. } \\
\text { Transformational leadership has a } \\
\text { significant and positive relationship } \\
\text { with quality of working life and a } \\
\text { negative relationship with burnout. }\end{array}$ \\
\hline \multirow[t]{2}{*}{$\begin{array}{l}\text { Kelloway } \\
\text { et al. } \\
(2012)\end{array}$} & Cross sectional & $\begin{array}{l}\text { Study } 1 \\
436 \text { Canadian } \\
\text { fieldworkers in a } \\
\text { large } \\
\text { telecommunication } \\
\text { organisation }\end{array}$ & 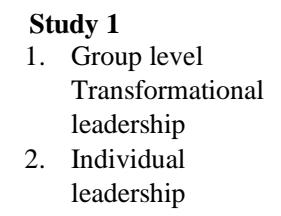 & $\begin{array}{l}\text { Psychological } \\
\text { wellbeing }\end{array}$ & Trust & None & $\begin{array}{l}\text { Employees trust in leadership fully } \\
\text { mediates the positive relationship } \\
\text { between Transformational leadership } \\
\text { and employee well-being }\end{array}$ \\
\hline & & $\begin{array}{l}\text { Study } 2 \\
269 \text { randomly } \\
\text { selected US } \\
\text { citizens working } \\
\text { for } 37.2 \text { hours } \\
\text { average } \\
\text { per week }\end{array}$ & $\begin{array}{ll}\text { Study } 2 \\
\text { 1. } & \text { Transformational } \\
& \text { leadership } \\
2 . & \text { Transactional } \\
& \text { leadership (TRL) }\end{array}$ & & & & $\begin{array}{l}\text { Replicated the mediated effects found } \\
\text { in Study } 1 \text { and outlined that } \\
\text { Transactional leadership negatively } \\
\text { affected well-being }\end{array}$ \\
\hline
\end{tabular}




\begin{tabular}{|c|c|c|c|c|c|c|c|}
\hline \multirow[b]{2}{*}{ Author } & \multirow[b]{2}{*}{ Study Design } & \multicolumn{4}{|c|}{ Variables } & \multirow[b]{2}{*}{ Moderators } & \multirow[b]{2}{*}{ Findings } \\
\hline & & Sample & Predictor Variables & $\begin{array}{l}\text { Outcome } \\
\text { Variables }\end{array}$ & Mediators & & \\
\hline $\begin{array}{l}\text { Krishnan } \\
(2012)\end{array}$ & Cross sectional & $\begin{array}{l}285 \text { Indian } \\
\text { managers of large } \\
\text { manufacturing } \\
\text { organisations }\end{array}$ & $\begin{array}{ll}\text { 1. } & \begin{array}{l}\text { Idealised } \\
\text { influence }\end{array} \\
\text { 2. } & \begin{array}{l}\text { Inspirational } \\
\text { motivation }\end{array} \\
\text { 3. } & \begin{array}{l}\text { Intellectual } \\
\text { stimulation }\end{array} \\
\text { 4. } & \begin{array}{l}\text { Individualised } \\
\text { consideration }\end{array}\end{array}$ & $\begin{array}{l}\text { Subjective } \\
\text { well-being }\end{array}$ & Empowerment & None & $\begin{array}{l}\text { Empowerment partially mediates the } \\
\text { relationship between Transformational } \\
\text { leadership and well-being }\end{array}$ \\
\hline $\begin{array}{l}\text { Liu et al. } \\
\text { (2010) }\end{array}$ & Cross sectional & $\begin{array}{l}745 \text { Chinese } \\
\text { employees (Beiging } \\
\text { \& Hong Kong) } \\
\text { working in the co- } \\
\text { operate sector }\end{array}$ & $\begin{array}{l}\text { Transformational } \\
\text { leadership }\end{array}$ & $\begin{array}{ll}\text { Employee well- } \\
\text { being: } \\
\text { 1. Job } \\
\text { satisfaction } \\
\text { 2. Perceived } \\
\text { work stress } \\
\text { 3. } & \begin{array}{l}\text { Stress } \\
\text { symptoms }\end{array}\end{array}$ & $\begin{array}{ll}\text { 1. } & \text { Trust } \\
\text { 2. } & \text { Self- } \\
& \text { efficacy }\end{array}$ & None & $\begin{array}{l}\text { Employees trust in the leader and self- } \\
\text { efficacy partially mediated the } \\
\text { influence of Transformational } \\
\text { leadership on job satisfaction and fully } \\
\text { mediated the influence of } \\
\text { Transformational leadership on } \\
\text { perceived work stress and symptoms. }\end{array}$ \\
\hline $\begin{array}{l}\text { Malik and } \\
\text { Tariq } \\
(2015)\end{array}$ & Cross sectional & $\begin{array}{l}180 \text { teachers in } \\
\text { Lahore ( } 2 \text { public } \\
\text { sector universities } 1 \\
\text { private sector } \\
\text { university) }\end{array}$ & $\begin{array}{l}\text { Transformational } \\
\text { leadership }\end{array}$ & $\begin{array}{l}\text { Psychological } \\
\text { wellbeing }\end{array}$ & Self-efficacy & None & $\begin{array}{l}\text { Self-efficacy fully mediated the } \\
\text { relationship between Transformational } \\
\text { leadership and well-being }\end{array}$ \\
\hline $\begin{array}{l}\text { McKee et } \\
\text { al. }(2011)\end{array}$ & Cross sectional & $\begin{array}{l}\text { Survey of } 178 \\
\text { employees at a non- } \\
\text { profit Canadian } \\
\text { organisation }\end{array}$ & $\begin{array}{l}\text { Transformational } \\
\text { leadership }\end{array}$ & $\begin{array}{l}\text { Employee well- } \\
\text { being }\end{array}$ & $\begin{array}{l}\text { Workplace } \\
\text { spirituality } \\
\text { (Meaningfulness, } \\
\text { sense of } \\
\text { community \& } \\
\text { shared values) }\end{array}$ & None & $\begin{array}{l}\text { The relationship between } \\
\text { Transformational leadership and well- } \\
\text { being was mediated by workplace } \\
\text { spirituality }\end{array}$ \\
\hline
\end{tabular}




\begin{tabular}{|c|c|c|c|c|c|c|c|}
\hline \multirow[b]{2}{*}{ Author } & \multirow[b]{2}{*}{ Study Design } & \multicolumn{4}{|c|}{ Variables } & \multirow[b]{2}{*}{ Moderators } & \multirow[b]{2}{*}{ Findings } \\
\hline & & Sample & Predictor Variables & $\begin{array}{l}\text { Outcome } \\
\text { Variables }\end{array}$ & Mediators & & \\
\hline $\begin{array}{l}\text { McMurray } \\
\text { et al. } \\
(2010)\end{array}$ & Cross sectional & $\begin{array}{l}43 \text { Australian non- } \\
\text { profit organisation } \\
\text { employees }\end{array}$ & $\begin{array}{l}\text { Transformational } \\
\text { leadership }\end{array}$ & $\begin{array}{l}\text { Employee well- } \\
\text { being }\end{array}$ & None & None & $\begin{array}{l}\text { A positive association between } \\
\text { Transformational leadership and well- } \\
\text { being }\end{array}$ \\
\hline $\begin{array}{l}\text { Munir et al. } \\
(2010)\end{array}$ & Longitudinal & $\begin{array}{l}188 \text { Danish } \\
\text { Eldercare workers }\end{array}$ & $\begin{array}{l}\text { Transformational } \\
\text { leadership }\end{array}$ & Well-being & None & None & $\begin{array}{l}\text { Transformational leadership was } \\
\text { associated with reduced depression in } \\
\text { followers. }\end{array}$ \\
\hline $\begin{array}{l}\text { Munir et al. } \\
\text { (2012) }\end{array}$ & Longitudinal & $\begin{array}{l}188 \text { staff working at } \\
\text { Danish elderly care } \\
\text { establishment }\end{array}$ & $\begin{array}{l}\text { Transformational } \\
\text { leadership }\end{array}$ & $\begin{array}{l}\text { Psychological } \\
\text { well-being }\end{array}$ & $\begin{array}{l}\text { Perception of } \\
\text { Work life } \\
\text { conflict }\end{array}$ & None & $\begin{array}{l}\text { Perceived work life conflict fully } \\
\text { mediated the relationship between } \\
\text { Transformational leadership and well- } \\
\text { being }\end{array}$ \\
\hline $\begin{array}{l}\text { Nielsen and } \\
\text { Munir } \\
(2009)\end{array}$ & Longitudinal & $\begin{array}{l}189 \text { staff working at } \\
\text { Danish elderly care } \\
\text { establishment }\end{array}$ & $\begin{array}{l}\text { Transformational } \\
\text { leadership }\end{array}$ & $\begin{array}{l}\text { Affective well- } \\
\text { being }\end{array}$ & Self-efficacy & None & $\begin{array}{l}\text { significant relationship between } \\
\text { transformational leadership and } \\
\text { affective well-being cross sectional } \\
\text { but not longitudinally. Self-efficacy } \\
\text { did not mediate the relationship } \\
\text { longitudinally }\end{array}$ \\
\hline $\begin{array}{l}\text { Nielsen, } \\
\text { Randall et } \\
\text { al. (2008) }\end{array}$ & Longitudinal & $\begin{array}{l}188 \text { health care } \\
\text { givers in a large } \\
\text { Danish local } \\
\text { government } \\
\text { department }\end{array}$ & $\begin{array}{l}\text { Transformational } \\
\text { leadership }\end{array}$ & $\begin{array}{l}\text { Psychological } \\
\text { well-being }\end{array}$ & $\begin{array}{l}\text { Work } \\
\text { characteristics: } \\
\text { Meaningfulness, } \\
\text { role clarity and } \\
\text { opportunities for } \\
\text { development }\end{array}$ & None & $\begin{array}{l}\text { Partial mediation between } \\
\text { Transformational leadership and } \\
\text { employee well-being at } t_{1} \text {, this partial } \\
\text { mediation was not found in } t_{2} \text { or over } \\
\text { time (full mediation in } t_{2} \text { ) }\end{array}$ \\
\hline
\end{tabular}




\begin{tabular}{|c|c|c|c|c|c|c|c|}
\hline \multirow[b]{2}{*}{ Author } & \multirow[b]{2}{*}{ Study Design } & \multicolumn{4}{|c|}{ Variables } & \multirow[b]{2}{*}{ Moderators } & \multirow[b]{2}{*}{ Findings } \\
\hline & & Sample & Predictor Variables & $\begin{array}{l}\text { Outcome } \\
\text { Variables }\end{array}$ & Mediators & & \\
\hline $\begin{array}{l}\text { Nielsen et } \\
\text { al. (2009) }\end{array}$ & Cross sectional & $\begin{array}{l}274 \text { Danish Elderly } \\
\text { care employees in } \\
\text { the local } \\
\text { government }\end{array}$ & $\begin{array}{l}\text { Transformational } \\
\text { leadership }\end{array}$ & $\begin{array}{l}\text { Psychological } \\
\text { well-being }\end{array}$ & $\begin{array}{l}\text { Team efficacy } \\
\text { and self-efficacy }\end{array}$ & None & $\begin{array}{l}\text { Both team and self-efficacy fully } \\
\text { mediated the relationship between } \\
\text { Transformational leadership and well- } \\
\text { being. }\end{array}$ \\
\hline $\begin{array}{l}\text { Nielsen and } \\
\text { Daniels } \\
(2012)\end{array}$ & Cross sectional & $\begin{array}{l}425 \text { Danish } \\
\text { Participants ( } 29 \\
\text { participants from } \\
\text { accountancy, } 27 \\
\text { elderly care groups) }\end{array}$ & $\begin{array}{ll}\text { 1. } & \text { Group level } \\
\text { Transformational } \\
\text { leadership } \\
\text { 2. } \\
\text { Differentiated } \\
\text { Transformational } \\
\text { leadership }\end{array}$ & $\begin{array}{l}\text { Well-being: } \\
\text { 1. Intention to } \\
\text { leave } \\
\text { 2. Job } \\
\text { satisfaction } \\
\text { 3. Burnout } \\
\text { 4. Sleep } \\
\text { quality } \\
\text { 5. Vitality }\end{array}$ & $\begin{array}{l}\text { Working } \\
\text { conditions } \\
\text { 1. Group and } \\
\text { individual level } \\
\text { meaningful } \\
\text { work } \\
\text { 2. Group and } \\
\text { Individual level } \\
\text { social support } \\
\text { 3. Group and } \\
\text { individual level } \\
\text { cohesion } \\
\text { 4. Group \& } \\
\text { Individual level } \\
\text { role clarity }\end{array}$ & None & $\begin{array}{l}\text { Work conditions fully mediated the } \\
\text { relationship between Group level } \\
\text { Transformational leadership, } \\
\text { Individual Transformational } \\
\text { leadership and features of wellbeing } \\
\text { (Only "vitality" partially mediated the } \\
\text { relationship between differentiated } \\
\text { Transformational leadership and } \\
\text { features of wellbeing) }\end{array}$ \\
\hline $\begin{array}{l}\text { Nielsen, } \\
\text { Yarker, et } \\
\text { al. (2008) }\end{array}$ & Cross sectional & $\begin{array}{l}447 \text { Danish } \\
\text { eldercare workers }\end{array}$ & $\begin{array}{l}\text { Transformational } \\
\text { leadership }\end{array}$ & Well-being & $\begin{array}{l}\text { Involvement, } \\
\text { influence and } \\
\text { meaning }\end{array}$ & None & $\begin{array}{l}\text { Involvement, influence and meaning } \\
\text { mediated the relationship between } \\
\text { Transformational leadership and } \\
\text { wellbeing, while meaningfulness } \\
\text { partially mediated the relationship }\end{array}$ \\
\hline $\begin{array}{l}\text { Salem } \\
(2015)\end{array}$ & Cross sectional & $\begin{array}{l}327 \text { employees in 5- } \\
\text { star hotels in Egypt. }\end{array}$ & $\begin{array}{l}\text { Transformational } \\
\text { leadership }\end{array}$ & Well-being & None & None & $\begin{array}{l}\text { A significant negative correlation } \\
\text { between Transformational leadership } \\
\text { and job stress and burnout }\end{array}$ \\
\hline $\begin{array}{l}\text { Schmidt et } \\
\text { al. (2014) }\end{array}$ & Cross sectional & $\begin{array}{l}320 \text { German White- } \\
\text { collar employees }\end{array}$ & $\begin{array}{l}\text { Transformational } \\
\text { leadership }\end{array}$ & $\begin{array}{l}\text { Psychological } \\
\text { strain }\end{array}$ & $\begin{array}{l}\text { Psychological } \\
\text { resources }\end{array}$ & None & $\begin{array}{l}\text { The relationship between } \\
\text { Transformational leadership and strain } \\
\text { was fully mediated by resources }\end{array}$ \\
\hline
\end{tabular}




\begin{tabular}{|c|c|c|c|c|c|c|c|}
\hline \multirow[b]{2}{*}{ Author } & \multirow[b]{2}{*}{ Study Design } & \multicolumn{4}{|c|}{ Variables } & \multirow[b]{2}{*}{ Moderators } & \multirow[b]{2}{*}{ Findings } \\
\hline & & Sample & Predictor Variables & $\begin{array}{l}\text { Outcome } \\
\text { Variables }\end{array}$ & Mediators & & \\
\hline $\begin{array}{l}\text { Sharifirad } \\
\text { (2013) }\end{array}$ & Cross sectional & $\begin{array}{l}583 \text { postgraduate } \\
\text { management } \\
\text { students in three top } \\
\text { universities in Iran } \\
\text { working either full- } \\
\text { time or part-time. }\end{array}$ & $\begin{array}{l}\text { Transformational } \\
\text { leadership }\end{array}$ & $\begin{array}{l}\text { Employee well- } \\
\text { being }\end{array}$ & $\begin{array}{l}\text { Empathetic } \\
\text { listening and } \\
\text { psychological } \\
\text { safety }\end{array}$ & None & $\begin{array}{l}\text { Empathetic listening and } \\
\text { psychological safety partially } \\
\text { mediated the relationship between } \\
\text { Transformational leadership and } \\
\text { employee wellbeing }\end{array}$ \\
\hline $\begin{array}{l}\text { Tafvelin et } \\
\text { al. (2011) }\end{array}$ & $\begin{array}{l}2 \text { wave } \\
\text { Longitudinal } \\
\text { panel study }\end{array}$ & $\begin{array}{l}\text { A stratified random } \\
\text { sample of } 136 \\
\text { Swedish social } \\
\text { service employees }\end{array}$ & $\begin{array}{l}\text { Transformational } \\
\text { leadership }\end{array}$ & $\begin{array}{l}\text { context free } \\
\text { affective well- } \\
\text { being }\end{array}$ & $\begin{array}{l}\text { Climate for } \\
\text { innovation as a } \\
\text { mediator }\end{array}$ & None & $\begin{array}{l}\text { Transformational leadership has no } \\
\text { direct effect on employee well-being } \\
\text { over time, however an indirect effect } \\
\text { was observed through positive climate } \\
\text { for innovation. Transformational } \\
\text { leadership was directly and positively } \\
\text { associated with well-being cross } \\
\text { section. }\end{array}$ \\
\hline $\begin{array}{l}\text { Walsh et al. } \\
\text { (2014) }\end{array}$ & Cross sectional & $\begin{array}{l}254 \\
\text { (Healthcare - 19\% } \\
\text { Financial - 19\% } \\
\text { Education - 19\% } \\
\text { Manufacturing - } \\
23 \% \\
\text { Retail - 20\%) }\end{array}$ & $\begin{array}{l}\text { Transformational } \\
\text { leadership }\end{array}$ & $\begin{array}{l}\text { Overall } \\
\text { psychological } \\
\text { health }\end{array}$ & $\begin{array}{l}\text { 1. Perceived } \\
\text { Procedural } \\
\text { Justice } \\
\text { 2. Psychological } \\
\text { empowerment }\end{array}$ & None & $\begin{array}{l}\text { Employees Perceived procedural } \\
\text { justice and psychological } \\
\text { empowerment fully mediated the } \\
\text { positive relationship between } \\
\text { Transformational leadership and } \\
\text { overall psychological health }\end{array}$ \\
\hline $\begin{array}{l}\text { Zwingmann } \\
\text { et al. } \\
(2014)\end{array}$ & Cross sectional & $\begin{array}{l}93,576 \text { subordinates } \\
\text { of large } \\
\text { multinational } \\
\text { organisation } \\
\text { (11.177 teams) } \\
\text { representing } 16 \\
\text { different countries } \\
\text { in Europe }\end{array}$ & $\begin{array}{l}\text { Transformational } \\
\text { leadership } \\
\text { (individual } \\
\text { and team) }\end{array}$ & $\begin{array}{l}\text { Wellbeing and } \\
\text { physical health }\end{array}$ & None & $\begin{array}{l}\text { Power } \\
\text { distance }\end{array}$ & $\begin{array}{l}\text { The results suggest that higher power } \\
\text { distance strengthens/moderates the } \\
\text { health promotion effects of } \\
\text { Transformational leadership. }\end{array}$ \\
\hline
\end{tabular}




\section{Liyanage}

\section{Findings}

This section will unveil the complex relationship between Transformational leadership and employee well-being, shedding light on the psychological mechanisms (i.e. the mediators and moderators) implicated in the said relationship. As will be discussed later in the section, several studies identified a link between transformational leadership and employee well-being, often pointing towards the complex and indirect relationship between the two constructs (e.g. Arnold et al., 2007; Bono et al., 2007; Corrigan et al., 2002). A common consensus among authors is that transformational leaders act as a buffer in the face of draining work demands. In other words, social support, feedback, trust and coaching provided by the leaders help to improve the employee's capability to deal with organisational demands like stress for instance (Offermann \& Hellmann, 1996; Sosik \& Godshalk, 2000).

By drawing out key elements of each paper, Table 1 synthesises the findings of the identified data base. Thereafter, the information has been mapped on a framework to illustrate the relationship between the two key concepts of Transformational leadership and employee well-being.

Based on the systematic review, a conceptual model was developed (as illustrated in Figure 2), which articulates the complex relationship between Transformational leadership and employee well-being. Psychological processes that mediated or partially mediated this relationship include: Meaningful work (Arnold et al., 2007; Nielsen \& Daniels, 2012; Nielsen, Randall et al., 2008), Empowerment (Krishnan, 2012; Walsh et al., 2014), Trust (Kelloway et al., 2012; Liu et al., 2010), Self-efficacy (Liu et al., 2010; Malik \& Tariq, 2015; Munir \& Nielesn, 2009; Nielsen et al., 2009), Psychological resources (Nielsen \& Daniels, 2012; Nielsen, Randall et al., 2008; Schmidt et al., 2014), Social support (Holstad et al., 2014), Procedural justice (Walsh et al., 2014), Quality of working life (Kara et al., 2013), Workplace spirituality (McKee et al., 2011), Perception of work-life conflict (Munir et al., 2012), Involvement and influence (Nielsen, Yarker et al., 2008), Empathetic listening and psychological safety (Sharifirad, 2013) and Climate for innovation (Tafvelin et al., 2011). The moderator variables as identified in the literature were Organisation commitment (Franke \& Felfe, 2011), Employee ambition (Holstad et al., 2014) and Power distance (Zwingmann et al., 2014). Among these mediators and moderators, it was noticed that the mediators - meaningful work, empowerment, trust, selfefficacy, personal resources, procedural justice - and the moderators - organisation commitment and power distance - have garnered greater attention in literature. Therefore, these mediators and moderators have been discussed in greater detail in 
the proceeding section. While the summary of the data is synthesised in Table 1, a detailed analysis of the results is illustrated in Figure 2.

Figure 2: The Relationship between Transformational Leadership and Employee Well-

\section{Being}

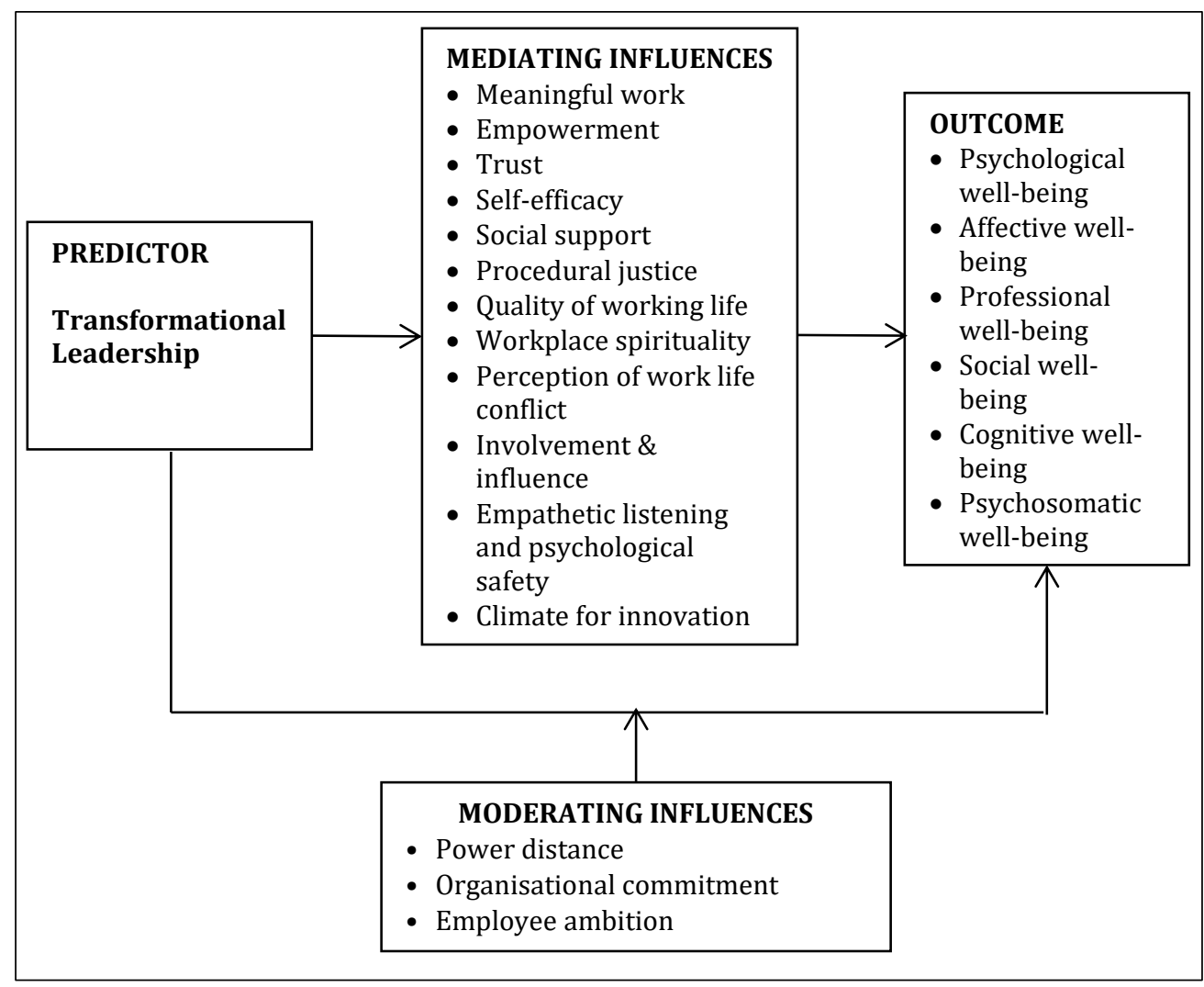

\section{Meaningful Work}

Out of the 30studies, 4 studies discuss the mediating role of meaningful work and broadly conceptualise meaningful work as "finding a purpose in life that is greater than the extrinsic outcomes of the work" (Arnold et al., 2007, p. 8).

Overall, all four studies were consistent with each other, where the results suggested that meaningful work mediates the relationship between Transformational leadership and employee well-being. However, the strength of the mediation varied between the studies (See Table 1). As per the results of the study by Arnold et al. (2007) positive Affective well-being was predicted by meaningful work $(\beta=0.49, p$ $<0.01$ ) and was not predicted by Transformational leadership, as the results were insignificant $(\beta=0.004, p<.05)$. Although, the study conducted by Nielsen and 
Daniels (2012) derived similar findings, the study suggests that perception of meaningful work mediates the relationship between differentiated Transformational leadership and well-being to a greater extent than group level perceptions of Transformational leadership. Here differentiated leadership refers to instances where the leaders adjust their behaviour to take account of individual differences of followers, therein providing "differentiated leadership" to the employees (Nielsen \& Daniels, 2012). Among the other work characteristics, meaningful work was found to mediate the relationship between well-being and almost all its antecedents.

The synthesis of literature suggests that the reason for such mediating effects is due to the unique traits of Transformational leadership discussed previously. For instance, through inspirational motivation, a Transformational leader constructs a clear vision and purpose for the organisation where the leader will innovatively illustrate how the employee's work will fit with the organisation's overall vision. As a result, followers will now obtain an increase in understanding of the shared goals and will attach more meaning in their daily work, in turn experiencing Eudaimonic well-being (Jung \& Sosik, 2002; Seltzer \& Bass, 1990). The larger impact of differentiated Transformational leadership on well-being through meaningful work is due to the individualised consideration attribute of differentiated Transformational leadership. Such leaders will not only provide personalised one-to-one training but will also help to analyse how an individual's personal goals are in alignment with the company's vision, which in turn will create more meaningful work. Through such differentiated Transformational leadership behaviour, employees may feel that they are more exposed to coaching and development opportunities than their colleagues, thus instigating a sense of meaning in their work (Neilsen \& Daniels, 2012).

Moreover, the followers may experience emotional contagion, where the passion expressed by the leader in articulating the company's purpose will be "caught" by the followers as well. Thereby, indirectly setting the scene for employees to attach more meaning to their work (Arnold et al., 2007). The fact that both studies (Arnold et al., 2007; Nielsen \& Daniels, 2012) suggested a complete mediation could be due to the presence of workers who have professions in relatively stigmatised occupations like funeral managers and directors. Such workers may actively attempt to redefine the work due to the stigma attached (Arnold et al., 2007).

\section{Empowerment}

Two studies in the review investigate the mediating impact of empowerment on the relationship between Transformational leadership and employee well-being 
(Kishnan, 2012; Walsh et al., 2014). Both these studies provide support for empowerment acting as a mediator. However, in the study conducted by Walsh et al. (2014), empowerment fully mediated the relationship between Transformational leadership and employee well-being ( $\beta=0.06$, NS), while the study by Krishnan (2012) predicts a partial mediation suggesting that a direct relationship between Transformational leadership dimension and employee well-being exists even when empowerment was considered [Idealised influence $(\beta=0.11, p<0.05)$; Inspirational motivation $(\beta=0.11, p<0.05)$; Intellectual stimulation $(\beta=0.10, p<0.05)$; Individualised consideration $(\beta=0.09, p<0.05)]$.

The overall evidence suggests that empowerment could be defined as having four key components namely, meaning, self-determination, impact and competence (Spreitzer, 1995). As discussed earlier Transformational leaders have the potential to encourage employees and create a sense of purpose for their followers, which in turn has a direct impact on empowerment (Spreitzer, 1995.). Employees who attach more meaning to their work will strive to complete a task and will take initiative to do so. In fact, the above findings suggest that empowerment influences well-being by enhancing the employees' ability to increase one's resources (Bakker \& Demerouti, 2007; Demerouti et al., 2001). This is because Transformational leaders place a high expectation on their followers and as a result such empowered workers go beyond their stipulated job roles to "get the job done." Employees in turn will experience further resource gain in terms of appreciation and recognition from superiors and colleagues. Such outcomes will contribute to a positive ripple effect where employees will feel more confident and self-efficacious, which is discussed in the next section. It is this spiral of resource acquisition that will lead to an improvement in employee's well-being (Walsh et al., 2014).

\section{Self-efficacy}

According to Bandura (1997, p.3) self-efficacy could be defined as "an individual's beliefs in one's capabilities to organise and execute the course of action required to produce given attainments". As self-efficacy alters the frame of mind of the employee and not the skill level, it is believed that Transformational leadership could instigate self-efficacious employees. Based on this assumption it was observed that four studies in the data base looked into the role of self-efficacy in mediating the relationship between Transformational leadership and employee well-being (Liu et al., 2010; Malik \& Tariq, 2015; Munir \& Nielsen, 2009; Nielsen et al., 2009). While all these studies predicted the role of self-efficacy as a mediator the magnitude of the impact of self-efficacy on employee well-being varied across the studies. The cross- 
sectional study conducted by Malik and Tariq (2015) suggest that Transformational leadership significantly influences well-being of teachers when self-efficacy was not considered $(\beta=0.26, p<0.05)$. However, when self-efficacy was added into the equation the direct relationship between the two variables became insignificant $(\beta=$ 1.3 , NS), suggesting that self-efficacy fully mediated the relationship between Transformational leadership and well-being, while self-efficacy continued to predict well-being $(\beta=0.46, p<0.05)$.

The results also hold for team-efficacy and self-efficacy, where the study conducted by Nielsen et al. (2009) within a healthcare setting identified that teamefficacy and self-efficacy fully mediated the relationship between Transformational leadership and well-being. While self-efficacy $(\beta=0.21, p<0.05)$ and team efficacy continued to predict employee well-being $(\beta=0.15, p<0.05)$.

While the study conducted by Liu et al. (2010 predicted similar results, where self-efficacy fully mediated the relationship between Transformational leadership and two indicators of well-being, (i.e. stress symptoms and perceived work stress) it only partly mediated the relationship between Transformational leadership and job satisfaction $(\beta=0.10, p<0.01))$. In this scenario the authors define satisfaction as satisfaction with the task, supervisor, co-workers, promotion and pay. As such it is reasonable to believe that such a direct impact exists as Transformational leaders may instigate a sense of satisfaction among the employees regarding the leader's behaviour, thereby establishing a direct relationship between Transformational leadership and the affective element of employee well-being (satisfaction).

The reason for such findings resides in the innate nature of Transformational leadership, a combination of individualised consideration and inspirational motivation where the leader will act as a mentor to employees. As leaders begin to understand the strengths and weaknesses of the employees, the Transformational leader will begin to play the role of an advisory suggesting how best employees could utilise resources to maximise performance. With time such leadership behaviour will fuel a sense of self confidence among the followers (Kirkpatrick \& Locke, 1996).

Such self-efficacious employees will engage in coping strategies during times of turbulence (Jex et al., 2001). Employees will take initiative to increase both personal and organisational resources, thereby resulting in a more engaged and less mentally drained employees (Bakker et al., 2003). Similarly, if an employee believes that they are contributing to a team where the colleagues are not only competent but also 
willing and capable to solve unanticipated problems, the said employee will take initiative to reciprocate such behaviour in order to feel part of the team, thereby, explaining the role of team efficacy in mediating the relationship. As such high efficacy is related to low job dissatisfaction, burnout, and increased state of employee well-being (Bandura, 1997; Judge \& Bono, 2000; Liu et al., 2010; Schyns \& von Collani, 2002).

\section{Procedural Justice}

Procedural justice concerns the "processes and symptoms through which decisions are made within an organisation" (Colquitt, 2001, p. 11). The crosssectional study conducted by Walsh et al. (2014) was the only research that attempted to identify if procedural justice acted as a mediator between Transformational leadership and well-being. The results suggest that well-being was predicted by procedural justice, where the indirect effect of Transformational leadership on psychological health occurred through procedural justice $(\beta=0.05, \mathrm{NS})$.

Through idealised influence, Transformational leaders ensure that the processes and systems implemented are fair. If the employee 'perceives' the outcomes of such processes to be fair they will experience less undue stress and strain about any potential injustices and will divert their energy and resources towards more productive activities. As employees experience less psychological strain, a state of well-being will be experienced (Schmidt et al., 2013; Walsh et al., 2014). Such fair leadership behaviour will help to engender trust among followers towards the leader, which in turn, will contribute to employee well-being as explained below.

\section{Trust}

Two studies in the selected database discussed the role of trust as a mediator between Transformational leadership and employee well-being (Kelloway et al., 2012; Liu et al., 2010). While both these studies established a mediating relationship the different contexts in which these studies were conducted contributed towards a variance in the magnitude of the results.

As outlined in Table 1, the study conducted by Kelloway et al. (2012) utilised two samples to ascertain the nature of the said relationship. The results of the first study, which involved no control variables, suggested that trust fully mediated the relationship between Transformational leadership and employee well-being $(\beta=0.05$, NS), even when certain elements of leadership personality were controlled trust continued to fully mediate the relationship. More specifically, this mediation was 
more significant for individual level leadership rather than group level leadership. This is in line with the findings of the study conducted by Nielsen and Daniels (2012) which suggested that the individual relationship with the Transformational leader was more predictive of employee well-being than group or shared perceptions of the leader. The strength of this study was increased by understanding the mediating effect of trust between Transactional leadership and employee well-being. Interestingly, the results indicated that Transformational leadership predicted trust $(\beta=0.29, p<0.05)$, while Transactional leadership did not $(\beta=-0.24$, NS) thereby, strengthening the aforesaid relationship of trust acting as a mediator between Transformational leadership and employee well-being.

The study conducted by Liu et al. (2010) had somewhat similar results. However, in contrast to the previous research, this study examined the mediating role of trust in the relationship between Transformational leadership and three antecedents of wellbeing (i.e. Job satisfaction, perceived work stress and stress symptoms). The results suggested that trust fully mediated the relationship between Transformational leadership and perceived work stress, Transformational leadership and stress symptoms, but only partially mediated the relationship between Transformational leadership and job satisfaction $(\beta=0.10, p<0.01)$.

The reason for such findings could be attributed to the idealised influence aspect of Transformational leadership. Such leaders are guided by what is moral and what is right for the employee. Hence, this aspect of a Transformational leader demonstrates the concern of leaders towards the employee's well-being. As the perceived threat of harm and stress caused by the superior is much less, followers begin to trust the leaders, thereby positively influencing employee well-being (Schabracq \& Cooper, 1996). As such, when employees trust their leaders the followers are 'willing to be vulnerable', and hence the implications on negative emotional and well-being could be less even during times of turmoil as they will 'trust' their leader to take the right course of action (Rousseau et al.,1998).

\section{Organisational Commitment}

The article by Franke and Felfe (2011) was the only study that discussed the role of organisational commitment as a moderator between Transformational leadership and well-being. In fact, the results suggested that the relationship between the Transformational leader's individualised consideration, idealised influence (attributed) and employee perceived strain are moderated by affective organisation commitment. Moreover, the study indicated that organisational commitment acted as 
a buffer during stressful conditions and also had a role to play in determining the degree of effect of Transformational leadership. For instance, if employees do not experience a strong connection/commitment to their organisation, the Transformational leader will help to reduce the perceived stress levels. In contrast, in the absence of organisational commitment, the perceived strain was high in employees when leaders do not display idealised influence and individual consideration.

The reason for these results is due to the fact that Transformational leaders have the ability to shed light during times of uncertainty. Low organisation commitment could be a symptom of times of turmoil, uncertainty and low job security. It is during such times that the impact of Transformational leadership could be seen. As Transformation leaders offer support, orientation and reliability, uncertainty and fear could be alleviated to a great extent (Frank \& Felfe, 2011) Therefore, if the organisational commitment is low, absence of Transformational leadership/poor leadership may only increase the perceived strain experienced by the employees.

\section{Power Distance}

The cross-cultural analysis of a large multinational organisation conducted by Zwingmann et al. (2014) was the only study that explored the role of power distance as a moderator between Transformational leadership and employee well-being.

Power distance is defined as "the extent to which society accepts the fact that power in organisations is distributed unequally" (Hofstede, 1980, p.8). Based on this definition the results of the study suggested that the relationship between Transformational leadership and employee well-being will be stronger for higher power distance cultures.

The reason for such findings is rooted in the cultural differences. Countries with high power distance have more red tape and greater levels of hierarchy than countries with low power distance. Such cultures prefer to be guided by their superiors where the goals and values expected are clearly delineated. Thus, such cultures value Transformational leadership behaviour as they are more willing to accept their leader's direction and vision. As employees place greater value on the resources provided by the Transformational leader, the positive cascading effect on employee well-being could also be significant. Therefore, well-being of followers residing in countries with higher power distance could be high (Hofstede, 1980; Zwingmann et al., 2014). 


\section{Job resources, Personal Resources and Demands}

As predicted by the Job Demand resource theory (JD-R), job resources (e.g. skill variety, task identity, task significance, autonomy and performance feedback) and personal resources (e.g. self-esteem, locus of control, self-efficacy) could contribute to employee well-being (Bakker \& Demerouti, 2007). While the conceptual model outlined in Figure 2 discusses certain job resources like empowerment, this section of the review looks to understand how such job resources in unison impact the relationship between Transformational leadership and employee well-being. In this endeavour four articles have been sourced to decode the relationship between Transformational leadership and employee well-being (Munir et al., 2011; Nielsen \& Daniels, 2012; Nielsen, Randall et al., 2008; Schmidt et al., 2013).

All four studies under review suggested that resources/work characteristics mediated the relationship between Transformational leadership and employee wellbeing. However, the extent of the mediation varied depending on the variables of the model under consideration. The longitudinal study conducted on 188 Danish healthcare givers by Nielsen, Randall et al. (2008) suggested that although in the short run, work characteristics only partially mediated the relationship between leadership and well-being, in the long run, work characteristics fully mediated the relationship. This raises the question whether, over time, Transformational leadership would result in well-being unless it influences changes in perceived work characteristics. However, there is some evidence that Transformational leadership at time one influenced well-being at time two through a relatively complex process. Such results are consistent with the longitudinal study conducted by Munir et al. (2011) which looked into the mediating impact of work-life conflict on Transformational leadership and well-being. The authors defined work-life conflict as job demands presented at home and at work where job and personal resources are insufficient to buffer the adverse impact of job demand. The study outlined that when perceived work-life conflict was not considered the relationship between Transformational leadership and well-being was significant $(\beta=0.20, p<0.05)$. When the perception of work life balance was included the relationship became insignificant $(\beta=0.12$, N.S), suggesting that positive perception of work-life balance fully mediated the relationship between Transformational leadership and well-being. This is because Transformational leaders through the distinct feature of individualised consideration attend to the followers' needs by playing the role of a mentor. Through instigating a supportive culture, Transformational leaders are likely to find solutions to work-life conflict, by providing social support and feedback. This will help employees to manage work-life conflicts contributing to a state of well-being (Grzywacz \& Marks, 2000; Wang \& Walumbwa, 2007). 
The two cross sectional studies conducted on job and personal resources suggested that resources fully mediated the relationship between Transformational leadership and most variables of well-being (Nielsen \& Daniels, 2012; Schmidt et al., 2014). Such results were not consistent with the longitudinal study by Nielsen, Randall et al (2008) which suggested that in the short run the resources partially mediated the relationship. For instance, the study conducted by Schmidt et al. (2013) on 320 German white-collar workers suggested that psychological resources fully mediated the relationship between Transformational leadership and psychological strain $(\beta=-0.33$, NS), while Transformational leadership predicted the employees' accumulation of resources $(\beta=0.61, p<0.01)$.

However, the results of this study conducted on Danish accountants and health care givers suggested that differentiated Transformational leadership had greater impact on well-being through the working conditions faced by the employees (Nielsen \& Daniels, 2012). In fact, 10 out of the 20 tests for mediation using differentiated Transformational leadership was significant compared to just 3 out of 20 for group level Transformational leadership (only one variable of well-being, vitality was partially mediated by differentiated Transformational leadership). The differentiated working conditions of social support, cohesion, meaningful work and role conflict mediated the relationship between Transformational leadership and at least one aspect of well-being criteria. This suggests that group level Transformational leadership impacts individual level well-being to a lesser extent than differentiated leadership. This is because special consideration from the leaders will improve the self-concept of the follower in turn positively influencing well-being (Nielsen \& Daniels, 2012).

The studies are in line with the JD-R and the Conservation of Resources (COR) theory which proposes that the individual strives to protect resources by building a strong 'resource reservoir' that could be used to combat undue circumstances of stress and burnout. In fact, the Transformational leader is by itself a resource, as Transformational leaders have the potential to indirectly influence psychological resources/working conditions by influencing the workload, job conditions and feedback rewards system (Hobfoll, 2001). As explained by the COR theory, employees strive to increase their resources thus, explaining the salient influence of the Transformational leader. These positive resource gains instigated by the Transformational leader will help to create resource canvas (e.g. provide meaning and role clarity) contributing to overall employee well-being. 


\section{The Direct Relationship between Transformational Leadership and Employee Well-being}

While the indirect effect of Transformational leadership on employee well-being was discussed earlier, this section will synthesise the evidence on the possible direct relationship between the two variables. From the 30studies, 13 identified a direct relationship between Transformational leadership and at least one component of wellbeing (Bono et al., 2007; Corrigan et al., 2002; Densten, 2005; Gill et al., 2010; Green et al., 2014; Jacobs et al., 2013; Krishnan, 2012; Liu et al., 2010; McMurray et al., 2010; Munir et al., 2010; Nielsen, Randall et al., 2008; Salem, 2015). This suggests that the impact of Transformational leadership on well-being is a complex process instigated by the leader through interrelated psychological processes.

The study conducted by Krishnan (2012) suggests that empowerment partially mediated the relationship between all features of Transformational leadership and well-being. This suggests that Transformational leadership directly influences wellbeing even in the presence of empowerment. However, all Transformational leadership dimensions do not predict employee well-being (Franke \& Felfe, 2011). While idealised influence (attributed) and individualised consideration had a significant relationship with employee well-being, idealised influence (behaviour) and inspirational motivation did not have a significant association. In fact, idealised influence (behaviour) $(\beta=0.09$, NS) and inspirational motivation $(\beta=0.12, \mathrm{NS})$ has a positive relationship with psychological strain, which is indicative of pseudo Transformational leadership or the 'dark side' of Transformational leadership (Bass, 1998).

Idealised influence (behaviour) as explained before is responsible for inspiring the followers through the morale and high-performance record of the Transformational leaders. As a result, employees may strive to emulate such leaders, strengthening employees' collective identity and fuelling them to take action to achieve the goals set. However, there is a possibility that the employees may forgo or neglect their personal needs and pursue the high standards set by the leader, which in turn may cause strain and overload (Seltzer et al., 1989). Similarly, inspirational motivation is responsible for motivating the follower to find meaning, purpose in life and achieve higher goals. In this endeavour, if the leader's expectation of the employee is high, subordinates may neglect other roles, and as a result, may be exposed to exhaustion and psychological strain. However, it is important to note that such potential negative effects are not intended by the Transformational leader. Transformational leaders act with good intention of influencing followers' 
performance levels in a positive manner. Despite these good intentions, employees may experience strain and overload. In contrast, idealised influence (attributed) revolves around the admirable attributes or qualities of the leader. Franke and Felfe (2011) suggest that it is this attributed charisma that is responsible for fuelling open communication and enhancing the follower's positive self-concept. This, combined with individualised consideration where leaders engage in a personalised relationship with the followers, will help to build a trustworthy and reliable relationship between the subordinate and the superior. As a result, employees will experience less strain even when external stressors are present. Thus, it is the Transformational leadership that is characterised by trust, recognition and empathy that would contribute to a higher state of well-being (van Dierendonck et al., 2014).

Moreover, the study by Zwingmann et al. (2014) suggests that the impact of Transformational leadership in well-being is universal. The study conducted on a multinational company that represented 16 countries suggested that while Transformational leadership significantly correlated with employee well-being in all of the sampled countries $(0.35<\mathrm{r}<0.50)$ it also correlated with the followers' health $(0.16<\mathrm{r}<0.34)$. However, the magnitude of the relationship differed based on sociocultural factors, business factors and the extent of the power distance as discussed before.

Based on the research findings, Zwingmann et al. (2014) also identified that a strong Transformational team climate was positively related to well-being and physical health in 7 of the 16 countries. Such results are in contrast to the study conducted by Kelloway et al. (2012) which suggests that individual level Transformational leadership is a greater predictor of well-being than group level Transformational leadership. The rationale for the latter is attributable to the positive impact of individualised consideration on employee self-concept and well-being. The difference in the results between the two studies could be due to differences in the contexts in which the studies were conducted.

Moreover, the longitudinal study conducted by Nielsen, Randall et al. (2008) suggested that work characteristics only partially mediated the relationship between Transformational leadership and well-being, indicating that a direct relationship between Transformational leadership and well-being exists in the short run $(\beta=0.13$, $p<0.01$ ). Most strikingly, this study found that a reverse relationship between wellbeing and Transformational leadership exists, where well-being at time 1 (i.e. short run) could predict Transformational leadership in time 2 (i.e. long run). Nielsen, Randall et al. (2008) point out that the employees experiencing sound well-being will 
be more energised and may in turn encourage their leaders to take action and would even question the status quo. As a result, employees' self-reported well-being may influence leadership behaviour, through a 'feedback loop' linking employee wellbeing and leader behaviour $(\beta=0.13, p<0.01)$.

\section{Discussion}

The main aim of this systematic literature review was to address the research question: "How does Transformational leadership influence employee well-being?" Applying the inclusion criteria to the data base resulted in 30 empirical papers to be incorporated in the systematic review. Thirty empirical papers are a surprisingly small number given the rising popularity of the concept of well-being in an occupational setting (Rewards \& Employee Benefits Association, 2018. However, a thorough search of the reference lists of the papers obtained from the initial scoping search provides a certain degree of confidence that all appropriate research was included in the review. In spite of this, the fact that only four databases were utilised suggest that pertinent articles related to the topic may have been omitted, which could the concern whether the conclusions arising from this review are based on the synthesis of all the available evidence.

All studies under consideration found that Transformational leadership predicted employee well-being. More specifically these studies attempted to decode this complex relationship by outlining the psychological processes that facilitated this relationship by testing the role of a number of mediators and moderators as identified earlier. This complex impact of Transformational leadership on employee well-being was observed across different organisational settings, industries, countries and participant characteristics, thereby, suggesting the positive implications of Transformational leadership on employee well-being, irrespective of contextual factors. Although the studies suggest that the psychological processes enable the impact of Transformational leadership on well-being, certain links had stronger associations with different indicators of well-being. As the context in which the studies were conducted varied it was difficult to draw inferences on the strongest or weakest link. Moreover, two of the cross-sectional studies suggested that differentiated Transformational leadership had a greater impact on well-being than group Transformational leadership (Nielsen \& Daniels, 2012; Keloway, 2012). Only one study conducted suggested otherwise, this is particularly due to the cross-cultural nature of the study conducted (Zwingmann et al., 2014). However, the overall result that Transformational leadership influences employee well-being through a 
psychological process is consistent with previous literature (e.g. Sivanathan et al., 2004; Walsh, 2001; Zohar, 2002).

The quality of studies reported in papers reviewed varied. Although a comprehensive quality assessment (Appendix 1) could not be done for all studies, an analysis of the data collection methods and the study design was conducted for all studies to ascertain the viability of the results obtained. Through quality analysis of the studies, it was found that all of the studies employed questionnaires as the primary research tool. All except one study used a universally reliable and tested tools to measure Transformational leadership. The exception being the study conducted by Liu et al. (2010), which was a scale developed purely for Chinese societies. However, to alleviate poor construct validity Liu et al. (2010) conducted a confirmatory factor analysis which portrayed an acceptable fit. The tools used to measure well-being were also reliable as a majority used reliable scales, which were validated using confirmatory factor analysis. Despite the different instruments used to operationalise well-being (e.g. Life scale of Pavot \& Diener, 1993 and Copenhagen Psychosocial Questionnaire) the findings were consistent, confirming that Transformational leadership influenced well-being through the distinct psychological processes outlined in the papers. Moreover, all the studies adopted a random sample of the population which was representative of the population selected. However, only six studies clearly explained the method of randomisation. While the response rates vary from $35 \%$ to $83 \%$, generalisability of the results were only mentioned in six articles and three out of the six suggested that it was not possible to generalise the findings as the sample sizes were relatively too small (Malik \& Tariq, 2015; Arnold et al., 2007). Moreover, all studies maintained the anonymity of the participant; this not only helped to enhance the response rates but also improved the ethical standing of the studies. In fact, when considering all studies, the strongest element was the reporting element, where each study carefully and cogently explained the objective, study design, sample size and main findings through robust statistical analysis.

However, the results of the studies should be interpreted with a degree of caution as there are some limitations that may undermine the accuracy of the findings. For instance, 24 out of the 30 studies used cross sectional research design, with which one cannot infer causality. There is a possibility that the hypothesised relationships may operate in the opposite direction to what is suggested. Another concern is the absence of agreement and clarity of the definition of well-being. Some studies operationalise well-being as an antecedent of attitudinal work constructs like psychological strain (e.g. Schmidt et al., 2013), subjective well-being (Krishnan et al., 2012), perceived work stress (e.g. Liu et al., 2010), intention to leave (e.g. Nielsen 
\& Daniels, 2012), physical health (e.g. Zwingmann et al., 2014), burnout (Nielsen \& Daniels, 2012), while other studies conceptualise and position well-being as a result of the above constructs (e.g. Kelloway, 2012; Munir et al., 2011; Neilsen, Yarker et al., 2008). Therefore, such varying views on the concept of well-being is an indication of the absence of a universally accepted definition, in turn, suggesting that the construct requires alignment and consensus between theory and empiricism within the well-being domain.

In line with the above argument Transformational leadership and well-being have been measured in many ways. While the multifactor Leadership questionnaire has dominated the assessment of Transformational leadership, indicators of the Copenhagen Psychosocial Questionnaire have been used in measuring well-being. Among these popular approaches other measurements of well-being have also emerged which suggests that there is a lack of comparability and replicability of the findings, due to the absence of consensus of the means of measurement of well-being and Transformational leadership. This as a result makes generalisability of the results improbable.

Another limitation is that six of the selected empirical studies used a short measure of Transformational leadership (Arnold et al., 2007; Krishnan, 2012; Liu et al., 2010; Nielsen \& Daniels, 2012; Walsh et al., 2014; Zwigmann et al., 2014). The reason for the adoption of a short measure of Transformational leadership was due to the belief that a lengthy questionnaire would adversely influence the reliability and validity of the measure as it may discourage participants to complete the questionnaire. The adoption of short measures would suggest that the six studies may have failed to capture all the dimensions of Transformational leadership. In fact, only two studies of all those reviewed explored all the dimensions of Transformational leadership (Franke \& Felfe, 2011; Krishnan, 2012). Therefore, the results of this systematic review could be limited in terms of implications for practice as the review did not extensively explore whether certain aspects of Transformational leadership drive the identified relationships.

Moreover, all the studies incorporated in the review employed self-reported data. As such there is a possibility that common method bias would have influenced the results. For instance, as the answers to the two constructs (Transformational leadership and well-being) were reported by the same person via the same medium (i.e. questionnaire), the correlations observed between the two variables could be due to the systematic error of using the same medium and rater (Podsakoff et al., 2003). 
Therefore, it is important to complement such methods with qualitative studies as well as studies that use multiple objective data or informants in order to obtain a holistic understanding of the impact of Transformational leadership on employee well-being.

In fact, the absence of qualitative methodologies utilizing methods such as indepth interviews limit the quality of the research, as such methods would allow the researcher to comprehend the experiences of the followers when dealing with a Transformational leader. The issue of lack of causality could also have been alleviated to a certain extent by the adoption of qualitative methods that inquire the impact of leader behaviour on follower's psychological health.

Although 7 of the 30 studies employed control variables, it is likely that there are other extraneous variables (e.g. work time preferences, working hours, and working conditions) which could influence the relationship between the two constructs. These, unaccounted factors may also have a moderating effect with Transformational leadership and may potentially contribute to different results (Wang \& Walumbwa, 2007). Moreover, to obtain a more holistic understanding of the impact of Transformational leadership on well-being, it would have been fitting to observe if a similar relationship exists between other types of leadership (e.g. transactional leadership) and employee well-being. Only one study, namely, Kelloway et al. (2012) observed the impact of transactional leadership on well-being. Thus, incorporating other types of leadership will help to obtain a holistic understanding of the impact of Transformational leadership on well-being.

The number of participants of studies reported in the papers reviewed ranged from 43 to 745, with only one cross cultural study conducted by Zwingman et al. (2014) utilizing a large sample incorporating 93,576 subordinates of a large multinational organisation. The relatively low sample size of the studies included reduces the power of the analysis, limiting the degree to which conclusions can be drawn. However, small sample sizes are not unusual in the realm of organisation behaviour.

Finally, due to certain publishing practices replication of studies is almost nonexistent. In consequence, a cumulative body of research findings have not surfaced that either support or refute most results. Thus, the quality in terms of the acceptability of the findings of these studies could be questioned. In fact, only one study, namely, Franke and Felfe (2011) replicated the findings of Seltzer et al. (1989) with the fivefactor concept of transformational leadership. 


\section{Strengths and Limitations}

The main strength of this review is its transparency and the systematic approach followed in collecting and presenting the evidence. For instance, it is believed that the use of the pre-determined search strategy complemented with appropriate Boolean operators helped to ensure that all studies pertaining to the subject of Transformational leadership and its impact on well-being were obtained (within the four data bases). Moreover, as the reference lists of all identified studies were screened for further articles, it could be stated that all potential studies exploring the selected constructs were obtained. In terms of assessing the quality of the studies a quality assessment form was devised. Due to time limitations a quality assessment was conducted only to five studies, however, a quality assessment in terms of the sample and data collection methods employed were perused for all studies, to ascertain the quality of the sampling techniques and data collection methods of the studies (Appendix 1). Apart from explicitly mentioning the inclusion and exclusion criteria, a flow diagram in the form of a PRISMA flow chart was adopted to delineate the steps adopted in conducting the review (Figure 1); this enhanced the reliability of this systematic review.

However, there are certain limitations that must be considered. Firstly, the review was limited to papers published in the English language. Moreover, only four data bases were researched, due to the inability to access certain databases. These factors may undermine the quality of the review due to the uncertainty if all relevant articles have been captured.

Based, on the quality assessment of the sampling techniques (Criteria 2 and 3 of Appendix 1), two articles were excluded as the data collection methods and assessment methods of the constructs were not clearly specified. Although these decisions to omit such papers were based on quality, it may have limited the range of the evidence considered. Moreover, due to time limitations, the complete quality assessment was not conducted on all studies (only Criteria $2 \& 3$, in Appendix 1 were assessed), and therefore, the quality of the articles and hence the generalisability of the articles cannot be guaranteed.

Finally, the review did not extend to include papers relating to engagement and flow, arguably these constructs are indicative of a state of employee well-being. Only studies that explicitly used scales that measure 'well-being' and constructs operationalised as well-being were considered. Therefore, other potential indications 
of well-being like engagement and flow were excluded. The decision to do so was due to theoretical reasons, as including such theoretical constructs would have stretched the concept of well-being too broadly. Such restrictions would have limited the number of articles which were included in the review.

\section{Conclusion}

Based on the systematic review conducted on the available empirical studies on Transformational leadership and employee well-being, a model was devised for understanding the interrelated connections between Transformational leadership and well-being (Figure 2). As per the synthesis of the literature, this model identified wellbeing as being operationalised including both negative (e.g. psychological strain) and positive indicators of well-being (e.g. positive state of mind); this approach is in line with renowned and established research practices (e.g. Warr, 1987).

Further, the model outlined that Transformational leadership influences employee well-being through a number of mediators, and also that there are several moderators influencing the relationship between these two variables. Although it is a possibility that certain mediators and moderators could have a stronger influence on the relationship between employee well-being and Transformational leadership, the results that surfaced through this review do not provide sufficient evidence to arrive at such conclusions, particularly due to the varying contexts in which these studies were conducted. Therefore, further research in this area is required. Moreover, it was also indicated that these psychological processes influenced one another. For instance, if an employee has the confidence in her/his abilities to complete a given task (e.g. self-efficacy), it is possible that this employee attaches more meaning to life and the work performed (e.g. meaningful work).

On the whole, this systematic review suggested that Transformational leadership predicted employee well-being through distinct psychological mechanisms, thereby drawing connections between two seemingly distinct concepts.

\section{Implications for Practice}

This review provides evidence that Transformational leadership predicts employee's well-being directly and indirectly through distinct psychological processes. Given the evidence that Transformational leadership is trainable would suggest that training leaders in Transformational leadership behaviours would instigate the psychological processes discussed in this review, which in turn would contribute towards employee well-being (Barling et al., 1996; Parry \& Sinha, 2005). 
The training should also focus on the varying impact of Transformational leadership, (Franke \& Felfe, 2011) thereby, instigating the leaders to be aware of their personal impact on employee well-being. The results also indicate that when evaluating the effectiveness of Transformational leadership, training organisations should attempt to understand the impact of the changed leader behaviour on employees through means of data gathering techniques like interviews and questionnaires, and not simply by understanding the change in leader's attitudes, behaviours and perceptions; because, unless the leader influences the employees' perceptions, it is unlikely that any impact on employee well-being would result (Munir et al., 2012; Nielsen, Randall et al., 2008; Walsh et al., 2014).

\section{Directions for Future Research}

Future research should address the limitations cited in the published articles; both methodological and conceptual limitations should be addressed. For instance, while future research should incorporate more longitudinal research designs it is important to conduct multiple wave studies incorporating multiple level analyses to refute any potential alternate explanations that could explain the relationship between Transformational leadership and employee well-being. For instance, future research should look into the effect of Transformational leadership in fostering group level employee well-being overtime and the role of the informal (Transformational) leader on individual and group level well-being must also be explored.

Moreover, a heavily under researched population is the stigmatised workers or the 'dirty workers' (Ashforth \& Kreiner, 1999; Hughes, 1951). Socially undesirable work such as the occupation of funeral directors cited in Arnold et al. (2007) is perceived to be degrading and stigmatised by society. Therefore, people who perform such roles could potentially experience low well-being. It would be interesting to observe the impact of Transformational leadership on alleviating the perceived stigma, and its role in fostering employee well-being. Empirical research is required to further ascertain this relationship.

\section{Acknowledgements}

I extend my thanks to Dr Hasuli Perera, Director of Studies and Senior Lecturer, Colombo Institute of Research and Psychology, for her comments that helped to finetune the review article. I would also like to thank the editors and reviewers for their useful and constructive comments which helped to improve the quality of the review. 


\section{Declaration of Conflicting Interests}

The authors declared no potential conflicts of interest with respect to the research, authorship, and publication of this article.

\section{References}

Arnold, K. A. (2017). Transformational leadership and employee psychological well-being: A review and directions for future research. Journal of Occupational Health Psychology, 22(3), 381-393. https://doi.org/10.1037/ocp0000062

Arnold, K. A., \& Connelly, C. E. (2015). Transformational leadership and psychological well-being: Effects on followers and leaders. In H. S. Leonard, R Lewis, A. M. Freedman, \& J. Passmore (Eds.), The Wiley-Blackwell handbook of the psychology of leadership, change, and organizational development (pp. 175-194). John Wiley \& Sons, Inc. https://doi.org/10.1002/9781118326404.ch9

Arnold, K. A., Turner, N., Barling, J., Kelloway, E. K., \& McKee, M. C. (2007). Transformational leadership and psychological well-being: The mediating role of meaningful work. Journal of Occupational Health Psychology, 12(3), 193203. https://doi.org/10.1037/1076-8998.12.3.193

Ashforth, B. E., \& Kreiner, G. E. (1999). "How can you do it?”: Dirty work and the challenge of constructing a positive identity. Academy of Management Review, 24(3), 413-434. https://www.jstor.org/stable/259134

Bakker, A. B., \& Demerouti, E. (2007). The Job Demands-Resources model: State of the art. Journal of Managerial Psychology, 22, 309-328. https://doi.org/10.1108/02683940710733115

Bakker, A. B., Demerouti, E., de Boer, E., \& Schaufeli, W. (2003). Job demands and job resources as predictors of absence duration and frequency. Journal of Vocational Behavior. 62(2). 341-356. https://doi.org/10.1016/S00018791(02)00030-1

Bandura, A. (1997). Self-efficacy: The exercise of control. Worth Publishers. Barling, J., Weber, T., \& Kelloway, E. K. (1996). Effects of transformational leadership training on attitudinal and financial outcomes: A field experiment. Journal of Applied Psychology, 81(6), 827-832. https://doi.org/10.1037/00219010.81.6.827

Bass, B. M. (1985). Transformational leadership and performance beyond expectation. Free Press.

Bass, B. M. (1998). The Ethics of Transformational Leadership. In J. B. Ciulla (Ed.), Ethics, the heart of leadership (pp. 169-192). Praeger. 
https://www.scirp.org/(S(351jmbntvnsjt1aadkposzje))/reference/ReferencesPap ers.aspx?ReferenceID $=2340931$

Bass, B. M., \& Avolio, B. J. (1993). Transformational leadership and organizational culture. Public Administration Quarterly, 112-121.

https://www.jstor.org/stable/40862298

Bass, B. M., \& Riggio, R. E. (2006). Transformational leadership ( $2^{\text {nd }}$ ed.). Psychology press.

Beauchamp, M. R., Barling, J., \& Morton, K. L. (2011). Transformational teaching and adolescent self-determined motivation, self-efficacy, and intentions to engage in leisure time physical activity: A randomised controlled pilot trial. Applied Psychology: Health and Well-Being, 3(2), 127-150. https://doi.org/10.1111/j.1758-0854.2011.01048.x

Boland, A., Cherry, G., \& Dickson, R. (2017). Doing a systematic review: A student's guide. Sage.

Bono, J. E., Foldes, H. J., Vinson, G., \& Muros, J. P. (2007). Workplace emotions: The role of supervision and leadership. Journal of Applied Psychology, 92(5), 1357-1367. https://doi.org/10.1037/0021-9010.92.5.1357

Briner, R. B., \& Denyer, D. (2012). Systematic review and evidence synthesis as a practice and scholarship tool. In D. M. Rousseau (Ed.), The Oxford handbook of evidence-based management (pp. 112-129). OXFORD University Press. https://www.oxfordhandbooks.com/view/10.1093/oxfordhb/9780199763986.00 1.0001/oxfordhb-9780199763986-e-7

Clarke, H. M., Arnold, K. A., \& Connelly, C. E. (2015). Improving follower wellbeing with transformational leadership. In S. Joseph (Ed.), Positive psychology in practice: Promoting human flourishing in work, health, education, and everyday life (p. 341-356). John Wiley \& Sons. https://doi.org/10.1002/9781118996874.ch21

Colquitt, J. A. (2001). On the dimensionality of organizational justice: A construct validation of a measure. Journal of Applied Psychology, 86(3), 386-400. https://doi.org/10.1037/0021-9010.86.3.386

Corrigan, P. W., Diwan, S., Campion, J., \& Rashid, F. (2002). Transformational leadership and the mental health team. Administration and Policy in Mental Health, 30, 97-108. https://doi.org/10.1023/A:1022569617123

Deci, E. L., \& Ryan, R. M. (2008). Hedonia, eudaimonia, and well-being: An introduction. Journal of Happiness Studies, 9(1), 1-11. https://doi.org/10.1007/s10902-006-9018-1

Demerouti, E., Bakker, A. B., Nachreiner, F., \& Schaufeli, W. B. (2001). The job demands-resources model of burnout. Journal of Applied Psychology, 86(3), 499-512. https://doi.org/10.1037/0021-9010.86.3.499 
Densten, I. L. (2005). The relationship between visioning behaviours of leaders and follower burnout. British Journal of Management, 16(2), 105-118. https://doi.org/10.1111/j.1467-8551.2005.00428.x

Franke, F., \& Felfe, J. (2011). How does Transformational leadership impact employees' psychological strain? Examining differentiated effects and the moderating role of affective organizational commitment. Leadership, 7(3), 295316. https://doi.org/10.1177/1742715011407387

Gill, A. S., Flaschner, A. B., \& Bhutani, S. (2010). The impact of transformational leadership and empowerment on employee job stress. Business and Economics Journal, 1, Article BEJ-3. https://astonjournals.com/bejvols.html

Green, A. E., Albanese, B. J., Shapiro, N. M., \& Aarons, G. A. (2014). The roles of individual and organizational factors in burnout among community-based mental health service providers. Psychological Services, 11(1), 4149. https://doi.org/10.1037/a0035299

Hobfoll, S. E. (2001). The influence of culture, community, and the nested-self in the stress process: Advancing conservation of resources theory. Applied Psychology, 50(3), 337-421. https://doi.org/10.1111/1464-0597.00062

Hofstede, G. (1980). Culture and organizations. International Studies of Management \& Organization, 10(4), 15-41. https://doi.org/10.1080/00208825.1980.11656300

Holstad, T. J., Korek, S., Rigotti, T., \& Mohr, G. (2014). The relation between transformational leadership and follower emotional strain: The moderating role of professional ambition. Leadership, 10(3), 269-288.

https://doi.org/10.1177/1742715013476083

Hughes, E. C. (1951). Work and the self. In J. H. Rohrer \& M. Sherif, Social psychology at the crossroads; The University of Oklahoma lectures in social psychology (p. 313-323). Harper.

International Labour Organization. (2010). Global wage report 2010/11: Wage policies in times of crisis.

https://www.ilo.org/global/publications/books/WCMS_145265/lang-en/index.htm

Jacobs, C., Pfaff, H., Lehner, B., Driller, E., Nitzsche, A., Stieler-Lorenz,B., Wasem, J., \& Jung, J. (2013). The influence of transformational leadership on employee well-being. Journal of Occupational and Environmental Medicine, 55(7), 772-778. https://doi.org/10.1097/JOM.0b013e3182972ee5

Jex, S. M., Bliese, P. D., Buzzell, S., \& Primeau, J. (2001). The impact of selfefficacy on stressor-strain relations: Coping style as an explanatory mechanism. Journal of Applied Psychology, 86(3), 401-409. https://doi.org/10.1037/00219010.86.3.401 
Judge, T. A., \& Bono, J. E. (2000). Five-factor model of personality and transformational leadership. Journal of Applied Psychology, 85(5), 751-765. https://doi.org/10.1037/0021-9010.85.5.751

Jung, D. I., \& Sosik, J. J. (2002). Transformational leadership in work groups: The role of empowerment, cohesiveness, and collective-efficacy on perceived group performance. Small Group Research, 33(3), 313-336. https://doi.org/10.1177/10496402033003002

Kara, D., Uysal, M., Sirgy, M. J., \& Lee, G. (2013). The effects of leadership style on employee well-being in hospitality. International Journal of Hospitality Management, 34, 9-18. https://doi.org/10.1016/j.ijhm.2013.02.001

Kelloway, E. K., Turner, N., Barling, J., \& Loughlin, C. (2012). Transformational leadership and employee psychological well-being: The mediating role of employee trust in leadership. Work \& Stress, 26(1), 39-55. https://doi.org/10.1080/02678373.2012.660774

Kirkpatrick, S. A., \& Locke, E. A. (1996). Direct and indirect effects of three core charismatic leadership components on performance and attitudes. Journal of Applied Psychology, 81(1), 36-51. https://doi.org/10.1037/0021-9010.81.1.36

Krishnan, V. R. (2012). Transformational leadership and personal outcomes: empowerment as mediator. Leadership \& Organization Development Journal, 33(6), 550-563. https://doi.org/10.1108/01437731211253019

Letts, L., Wilkins, S., Law, M., Stewart, D., Bosch, J., \& Westmorland, M. (2007). Guidelines for critical review form: Qualitative studies (Version 2). McMaster University Occupational Therapy Evidence-Based Practice Research Group. https://www.semanticscholar.org/paper/Guidelines-for-critical-review-formQualitative-Letts-Wilkins/74513fac0424cd8210f9fc9577d3e9bc721bd06c

Liu, J., Siu, O. L. \& Shi, K. (2010). Transformational leadership and employee well-being: The mediating role of trust in the leader and self-efficacy. Applied Psychology, 59(3),.454-479. https://doi.org/10.1111/j.1464-0597.2009.00407.x

Long, A. F., Godfrey, M., Randall, T., Brettle, A. J., \& Grant, M. J. (2002). Developing Evidence Based Social Care Policy and Practice. Part 3: Feasibility of Undertaking Systematic Reviews in Social Care. University of Leeds (Nuffield Institute for Health); University of Salford (Health Care Practice R\&D Unit). http://usir.salford.ac.uk/id/eprint/13071/

Malik, S. Z., \& Tariq, S, (2015). Relationship of transformational leadership, psychological well-being and self efficacy: A mediation analysis in university faculty. Journal of Quality and Technology Management, 9(2), 93-109. http://pu.edu.pk/images/journal/iqtm/PDF-FILES/05Relationship\%20of\%20Transformational_v_xi_II_Dec_2015.pdf 
Medley, F., \& Larochelle, D. R. (1995). Transformational leadership and job satisfaction. Nursing Management, 26(9), 64JJ-64LL. https://doi.org/10.1097/00006247-199509000-00017

McKee, M. C., Driscoll, C., Kelloway, E. K., \& Kelley, E. (2011). Exploring the linkages among transformational leadership, workplace spirituality and wellbeing in health care workers. Journal of Management, Spirituality \& Religion, 8(3), 233-255. https://doi.org/10.1080/14766086.2011.599147

McMurray, A. J., Pirola-Merlo, A., Sarros, J. C., \& Islam, M. M. (2010). Leadership, climate, psychological capital, commitment, and wellbeing in a non-profit organization. Leadership \& Organization Development Journal, 31(5), 436-457. https://doi.org/10.1108/01437731011056452

Munir, F., \& Nielsen, K. (2009). Does self-efficacy mediate the relationship between transformational leadership behaviours and healthcare workers' sleep quality? A longitudinal study. Journal of Advanced Nursing, 65(9), 1833-1843. https://doi.org/10.1111/j.1365-2648.2009.05039.x

Munir, F., Nielsen, K., \& Carneiro, I. G. (2010). Transformational leadership and depressive symptoms: A prospective study. Journal of Affective Disorders, 120, 235-239. https://doi.org/10.1016/j.jad.2009.03.020

Munir, F., Nielsen, K., Garde, A. H., Albertsen, K., \& Carneiro, I. G. (2012). Mediating the effects of work-life conflict between transformational leadership and health-care workers' job satisfaction and psychological well-being. Journal of Nursing Management, 20(4), 512-521. https://doi.org/10.1111/j.13652834.2011.01308.x

Nielsen, K., \& Daniels, K. (2012). Does shared and differentiated transformational leadership predict followers' working conditions and well-being? The Leadership Quarterly, 23(3), 383-397. https://doi.org/10.1016/j.leaqua.2011.09.001

Nielsen, K., Randall, R., Yarker, J., \& Brenner, S.-O. (2008). The effects of transformational leadership on followers' perceived work characteristics and psychological well-being: A longitudinal study. Work \& Stress, 22(1), 16-32. https://doi.org/10.1080/02678370801979430

Nielsen, K., Yarker, J., Brenner, S.-O., Randall, R. \& Borg, V. (2008). The importance of transformational leadership style for the well-being of employees working with older people. Journal of Advanced Nursing, 63(5), 465-475. https://doi.org/10.1111/j.1365-2648.2008.04701.x

Nielsen, K., Yarker, J., Randall, R., \& Munir, F., (2009). The mediating effects of team and self-efficacy on the relationship between transformational leadership, and job satisfaction and psychological well-being in healthcare professionals: A 
cross-sectional questionnaire survey. International Journal of Nursing Studies, 46(9), 1236-1244. https://doi.org/10.1016/j.ijnurstu.2009.03.001

Offermann, L. R., \& Hellmann, P. S. (1996). Leadership behavior and subordinate stress: A 360" view. Journal of Occupational Health Psychology, 1(4), 382390. https://doi.org/10.1037/1076-8998.1.4.382

Organisation for Economic Co-operation and Development (2011). OECD employment outlook 2011. OECD Publishing. https://doi.org/10.1787/empl_outlook-2011-en

Page, K. M., \& Vella-Brodrick, D. A. (2009). The 'what', 'why' and 'how' of employee well-being: A new model. Social Indicators Research, 90, 441-458. https://doi.org/10.1007/s11205-008-9270-3

Parry, K. W., \& Sinha, P. (2005). Researching the trainability of transformational organisational leadership. Human Resource Development International, 8(2), 165-183. https://doi.org/10.1080/13678860500100186

Pavot, W., \& Diener, E. (1993). Review of the satisfaction with life scale. Psychological Assessment, 5(2), 164-172.

Pearce, C. L., \& Conger, J. A. (2003). Shared leadership: Reframing the hows and whys of leadership. SAGE Publications.

Podsakoff, P. M., MacKenzie, S. B., Lee, J-Y., \& Podsakoff, N. P. (2003). Common method biases in behavioral research: A critical review of the literature and recommended remedies. Journal of Applied Psychology, 88(5), 879-903. https://doi.org/10.1037/0021-9010.88.5.879

Popay, J., Roberts, H., Sowden, A., Petticrew, M., Arai, L., Rodgers, M., \& Duffy, S. (2006). Guidance on the conduct of narrative synthesis in systematic reviews (Version1). ESRC methods programme. http://citeseerx.ist.psu.edu/viewdoc/download?doi=10.1.1.178.3100\&rep=rep1 \&type $=$ pdf

Reward \& Employee Benefits Association \& Punter Southall Health \& Protection (2018). Employee wellbeing research 2018. https://reba.global/files/document/171/1521138370_REBAEmployeeWellbeing Research2018ESV_web.pdf

Robertson, I. T., \& Cooper, C. L. (2010). Full engagement: The integration of employee engagement and psychological well-being. Leadership \& Organization Development Journal, 31(4), 324-336. https://doi.org/10.1108/01437731011043348

Rousseau, D. M. (1998). Journal of Organizational Behavior, 19(S1), 665-671. https://doi.org/10.1002/(SICI)1099-1379(1998)19:1+<665::AIDJOB972>3.0.CO;2-X 
Salem, I. E-B. (2015). Transformational leadership: Relationship to job stress and job burnout in five-star hotels. Tourism and Hospitality Research, 15, 240-253. https://doi.org/10.1177/1467358415581445

Seltzer, J., \& Bass, B. M. (1990). Transformational leadership: Beyond initiation and consideration. Journal of Management, 16(4), 693-703. https://doi.org/10.1177/014920639001600403

Seltzer, J., Numerof, R. E., \& Bass, B. M. (1989). Transformational leadership: Is it a source of more burnout and stress? Journal of Health and Human Resources Administration, 12(2), 174-185. http://www.jstor.org/stable/25780396

Schabracq, M. J., \& Cooper, C. L. (2000). The changing nature of work and stress. Journal of Managerial Psychology, 15(3), 227-241. https://doi.org/10.1108/02683940010320589

Schmidt, B., Loerbroks, A., Herr, R., Litaker, D., Wilson, M., Kastner, M., \& Fischer, J. (2014). Psychosocial resources and the relationship between transformational leadership and employees' psychological strain. Work Behaviour, 49(2), 315-324. https://doi.org/10.3233/WOR-131713

Schyns, B., \& von Collani, G. (2002). A new occupational self-efficacy scale and its relation to personality constructs and organizational variables. European Journal of Work and Organizational Psychology, 11(2), 219-241. https://doi.org/10.1080/13594320244000148

Sharifirad, M. S. (2013). Transformational leadership, innovative work behavior, and employee well-being. Global Business Perspectives, 1(3), 198-225. https://doi.org/10.1007/s40196-013-0019-2

Sivanathan, N., Arnold, K.A., Turner, N., \& Barling, J. (2004). Leading well: Transformational leadership and well-being. In P. A. Linley \& S. Joseph (Eds.), Positive psychology in practice (p. 241-255). John Wiley \& Sons.

Sofarelli, D., \& Brown, D. (1998). The need for nursing leadership in uncertain times. Journal of Nursing Management, 6(4), 201-207. https://doi.org/10.1046/j.1365-2834.1998.00075.x

Sosik, J. J., \& Godshalk, V. M. (2000). Leadership styles, mentoring functions received, and job-related stress: A conceptual model and preliminary study. Journal of Organizational Behavior, 21(4), 365-390. https://doi.org/10.1002/(SICI)1099-1379(200006)21:4<365::AIDJOB14>3.0.CO;2-H

Spreitzer, G. M. (1995). Psychological empowerment in the workplace: Dimensions, measurement, and validation. Academy of Management Journal, 38(5), 1442-1465. https://doi.org/10.5465/256865

Tafvelin, S., Armelius, K., \& Westerberg, K. (2011). Toward understanding the direct and indirect effects of transformational leadership on well-being: A 
longitudinal study. Journal of Leadership \& Organizational Studies, 18(4), 480-492. https://doi.org/10.1177/1548051811418342

Thyer, G. L. (2003). Dare to be different: Transformational leadership may hold the key to reducing the nursing shortage. Journal of Nursing Management, 11(2), 73-79. https://doi.org/10.1046/j.1365-2834.2002.00370.x

Trofino, A. J. (2000). Transformational leadership: Moving total quality management to world-class organizations. International Nursing Review, 47(4), 232-242. https://doi.org/10.1046/j.1466-7657.2000.00025.x

Turner, N., Barling, J., Epitropaki, O., Butcher, V., \& Milner, C. (2002). Transformational leadership and moral reasoning. Journal of Applied Psychology, 87(2), 304-311. https://doi.org/10.1037/0021-9010.87.2.304

van Dierendonck, D., Stam, D. A., Boersma, P., De Windt, N., \& Alkema, J. (2014). Same difference? Exploring the differential mechanisms linking servant leadership and transformational leadership to follower outcomes. The Leadership Quarterly, 25(3), 544-562. https://doi.org/10.1016/j.leaqua.2013.11.014

Walsh, M., Dupré, K., \& Arnold, K. A. (2014). Processes through which transformational leaders affect employee psychological health. German Journal of Human Resource Management, 28(1-2), 162-172. https://www.jstor.org/stable/24332807

Zwingmann, I., Wegge, J., Wolf, S., Rudolf, M., Schmidt, M., \& Richter, P. (2014). Is transformational leadership healthy for employees? A multilevel analysis in 16 nations. German Journal of Human Resource Management, 28(1-2), 24-51. https://doi.org/10.1177/239700221402800103

\section{Appendix 1: Quality Assessment Form: Quantitative Studies}

\begin{tabular}{|c|c|}
\hline Review area & Question \\
\hline Study overview & $\begin{array}{l}\text { What is the purpose of the study? } \\
\text { What are the aims of the study? } \\
\text { What are the strengths and weakness of the study and theory? }\end{array}$ \\
\hline Design & What was the research design adopted? \\
\hline Sample & $\begin{array}{l}\text { Were the criteria for inclusion in the sample clearly defined? } \\
\text { Was the sample described in detail? (e.g. What are the characteristics } \\
\text { of the sample) } \\
\text { How was the sampling done? } \\
\text { Is the sample size justified? } \\
\text { Were the study subjects and the setting described in detail? }\end{array}$ \\
\hline
\end{tabular}




\begin{tabular}{|c|c|}
\hline Review area & Question \\
\hline Outcomes & $\begin{array}{l}\text { Were the outcome measures reliable? } \\
\text { Were the outcome measures valid? }\end{array}$ \\
\hline Intervention & $\begin{array}{l}\text { Were the variables to be tested clearly identified? } \\
\text { Were objective criteria used for measurement of a condition? } \\
\text { Were confounding factors identified? } \\
\text { Were the strategies to deal with the confounding factors stated? }\end{array}$ \\
\hline Results & $\begin{array}{l}\text { Were the outcomes measured in a valid and reliable way? } \\
\text { Were the results reported in terms of statistical significance? } \\
\text { Were any dropouts reported? }\end{array}$ \\
\hline $\begin{array}{l}\text { Conclusions and } \\
\text { implications }\end{array}$ & $\begin{array}{l}\text { What did the study conclude? } \\
\text { What are the implications of the results in a practical organization } \\
\text { setting? }\end{array}$ \\
\hline
\end{tabular}

Source: Adapted from Long et al. (2002) 